\title{
Electron Localization of Anions Probed by Nitrile
}

\section{Vibrations}

Tomoyasu Mani, * David C. Grills, Marshall D. Newton, and John R. Miller

Chemistry Department, Brookhaven National Laboratory, Upton NY 11973-5000, United States

\section{Corresponding Author}

*tmani@bnl.gov 
ABSTRACT. Localization and delocalization of electrons is a key concept in chemistry, and is one of the important factors determining the efficiency of electron transport through organic conjugated molecules, which have potential to act as "molecular wires." This in turn, substantially influences the efficiencies of organic solar cells and other molecular electronic devices. It is also necessary to understand the electronic energy landscape and the dynamics of electrons through molecular chains that govern their transport capabilities in one-dimensional conjugated chains so that we can better define the design principles of conjugated molecules for their applications. We show that nitrile $v(\mathrm{C} \equiv \mathrm{N})$ vibrations respond to the degree of electron localization in nitrile-substituted organic anions by utilizing time-resolved infrared (TRIR) detection combined with pulse radiolysis. Measurements of a series of aryl nitrile anions allow us to construct a semi-empirical calibration curve between the changes in the $v(\mathrm{C} \equiv \mathrm{N})$ IR shifts and the changes in the electronic charges from the neutral to the anion states in the nitriles; more electron localization in the nitrile anion results in larger IR shifts. Furthermore, the IR linewidth in anions can report a structural change accompanying changes in electronic density distribution. Probing the shift of the nitrile $v(\mathrm{C} \equiv \mathrm{N})$ IR vibrational bands enables us to determine how the electron is localized in anions of nitrile-functionalized oligofluorenes, considered as organic mixed-valence compounds. We estimate the diabatic electron transfer distance, electronic coupling strengths, and energy barriers in these organic mixed-valence compounds. The analysis reveals a dynamic picture, showing that the electron is moving back and forth within the oligomers with a small activation energy of $\leq k_{\mathrm{B}} T$, likely controlled by the movement of dihedral angles between monomer units. Implications for the electron transport capability in "molecular wires" are discussed. 


\section{Introduction}

Localization and delocalization of electrons is key to determining the efficiency of electron transport through organic conjugated molecules, known as "molecular wires." They are in turn among the important parameters that affect the efficiencies of organic solar cells and other molecular electronics applications. ${ }^{1}$ More spatially delocalized excitons and polarons are generally thought to move faster through conjugated molecules. ${ }^{2,3}$ Unlike for acenes and other small polycyclic aromatic hydrocarbons, the degree of electron delocalization of long oligomers and polymers is difficult to define and measure experimentally. It is often defined by the number of monomer units an extra electron occupies, ${ }^{4}$ which can be considered as the edge-to-edge length $\left(l_{\mathrm{del}}\right)$. Usually, $l_{\text {del }}$ is determined by optical measurements ${ }^{4}$ or redox potentials of oligomers. ${ }^{5,6} l_{\text {del }}$ of a limited number of polymers have been measured and they range from 2 to 6 nm. ${ }^{4,6,7}$ While $l_{\text {del }}$ has been used previously, its physical definition is not obvious and an alternative measure of electron delocalization is desired.

In a closely related phenomenon, an electron transfer process from donor to acceptor molecules sometimes results in a partial charge transfer, producing a charge-transfer state or an exciplex. Understanding charge-transfer states is critical since knowledge of the degree of charge separation and delocalization is important for controlling the efficiency of organic photovoltaics. Both the degree of electron localization and the degree of charge transfer reflect the electronic density distribution among the molecules, which is not easy to measure experimentally. It is known that electrons and holes in conjugated molecules exhibit intense IR bands, ${ }^{8,9}$ especially $v(\mathrm{C}=\mathrm{C})$ bands, and that their intensities are dependent upon delocalization of charges. ${ }^{9}$ Infrared (IR) spectroscopy has previously been used to discern charge transfer states by means of spectral shifts of specific vibrational bands such as $v(\mathrm{C}=\mathrm{O})$ bands ${ }^{10-15}$ and $v(\mathrm{C}=\mathrm{C})$ bands. ${ }^{16}$ In a similar 
manner, we postulate that we can measure the degree of electron localization by spectral shifts of specific vibrational bands.

Spatial delocalization and the degree of charge transfer are measures of "static" properties. In addition to these parameters, it is necessary to understand the electronic energy landscape and dynamics throughout molecular chains that govern electron transport capabilities in one-dimensional "molecular wires" so that we can better define the design principle of conjugated molecules or more generally, $\pi$-systems for organic photovoltaics or other applications. This information is often difficult to attain in long conjugated polymers.

Mixed-valence compounds give access to dynamic parameters such as rate constants, diabatic electronic coupling $\left(H_{\mathrm{ab}}\right)$, and activation energies or energy barriers $\left(\Delta G^{\ddagger}\right)$ for intramolecular electron transfer. ${ }^{17-19}$ They are molecular systems that contain two redox centers in different oxidation states $\left(\mathrm{M}^{\mathrm{n}}\right.$ and $\mathrm{M}^{\mathrm{n}+1}$, where $\mathrm{n}$ is an integer) usually attached symmetrically to a bridge. Among families of mixed-valence compounds, organic mixed-valence compounds have been extensively studied over the last two decades because of their potential use in organic light emitting diodes (OLED), organic photovoltaics, and other applications. ${ }^{20}$ Moreover, the studies of these systems can give us fundamental knowledge of electron and hole transfer processes in organic molecules. $^{21,22}$

Here, we illustrate how studies of rationally-designed oligomeric molecules can provide insight into the static and dynamic properties of electrons in long conjugated polymers that are often difficult to study. The goal of this paper is two-fold. In the first section, making use of pulse radiolysis coupled with time-resolved infrared (TRIR) detection, we report large shifts of $v(\mathrm{C} \equiv \mathrm{N})$ IR vibrations for the anions of a series of nitrile-functionalized aryl compounds, the magnitudes of which depend on the degree of electron localization. Pulse radiolysis experiments 
enable us to inject a single electron into a molecule, rapidly producing a free anion without ion pairing, which is monitored in a time-resolved fashion. We construct a semi-empirical calibration curve relating the changes in the $v(\mathrm{C} \equiv \mathrm{N})$ IR shifts to the computed charges of the nitrile, allowing us to estimate partial charge distributions in unknown aryl nitriles. We also estimate the degree of electron delocalization in anions by using second moments of state densities as an alternative measure to the previously used $l_{\mathrm{del}}$. In the second section of the paper, we present a study of dinitrile-functionalized oligofluorenes that can be considered as organic mixed-valence compounds. We introduce a new method, called the IR-CHARGE method, to determine the adiabatic electron transfer distance using the IR-derived information based on the results of the first section. Electronic couplings and other electron transfer parameters are determined by the Generalized Mulliken-Hush (GMH) theory using the IR-CHARGE method as well as a wellestablished optical spectroscopy method, and in addition by a Koopmans' Theorem (KT) calculation. Our analysis delineates a dynamic picture of electron movement in anions of organic conjugated molecules, giving physical insight into electron movements in one-dimensional “molecular wires.”

\section{Experimental Methods}

General Information. All solvents and reagents used were obtained from standard commercial sources and used as received unless otherwise noted. Silica gel (Sigma-Aldrich, pore size $60 \AA \AA$, 70-230 mesh) was used for column chromatography. FTIR spectra were recorded on a Thermo Nicolet Nexus 670 FTIR spectrometer using a cell equipped with $\mathrm{CaF}_{2}$ windows. ${ }^{1} \mathrm{H}$ and ${ }^{13} \mathrm{C}$ NMR spectra were recorded with a Bruker Avance III spectrometer operating at 400.16 and 100.62 MHz, respectively. UV-vis-NIR absorption spectra were recorded with a Cary 5 
spectrophotometer (Varian). For spectroscopic measurements other than NMR, samples were prepared under an inert atmosphere condition. Tetrahydrofuran (THF) was purified with a Vacuum Atmospheres Solvent Purifier System installed inside an inert atmosphere glovebox.

Synthesis. The complete synthetic scheme, synthetic procedures, and the characterizations of new compounds are described in the Supporting Information. $\mathbf{F}_{\mathbf{1}} \mathbf{C N}^{23}$ was synthesized as previously reported. Optical absorption spectra of the neutral species are shown in Supporting Information Figure S1.

Chemical Reduction with Sodium Biphenyl. Chemical reduction was performed with sodium biphenyl in THF in the presence of excess cryptand C222 (c 12 mM), in a similar manner to the established procedure. ${ }^{7}$ Cryptands C222 are used to encapsulate $\mathrm{Na}^{+}$counterions to reduce the effects of ion pairing. ${ }^{24}$ The experiments were performed under an inert argon atmosphere.

Electrochemistry. Cyclic voltammetry measurements were conducted with a BAS 100B electrochemical analyzer (Bioanalytical Systems) in a standard three-electrode cell consisting of a $3 \mathrm{~mm}$ glassy carbon disc working electrode, a platinum wire counter electrode, and a reference electrode consisting of $\mathrm{Ag} / \mathrm{AgNO}_{3}$ in THF solution with $0.1 \mathrm{M}$ tetrabutylammonium hexafluorophosphate $\left(\mathrm{TBA}^{+} \mathrm{PF}_{6}^{-}\right)$isolated from the main compartment by a Vycor frit. Potentials are reported $v s \mathrm{Fc}^{+/ 0}$; ferrocene was added after the measurements.

Pulse Radiolysis. Pulse radiolysis experiments were performed at the Laser-Electron Accelerator Facility (LEAF) at Brookhaven National Laboratory. For UV-vis-NIR transient absorption detection, the experiments were performed as described before, ${ }^{25}$ using a quartz cell with a pathlength of $0.5 \mathrm{~cm}$. For TRIR detection, a detailed description of the experimental setup 
is given elsewhere. ${ }^{26}$ A home-built, airtight IR solution flow cell was used (1.10 mm pathlength), with $0.35 \mathrm{~mm}$ thick $\mathrm{CaF}_{2}$ windows. Continuous wave external-cavity quantum cascade lasers (Model 21047-MHF and Model 21043-MHF, Daylight Solutions, Inc.) were used as the IR probe source. The time resolution is limited to $\sim 40$ ns in the current setup. Samples were dissolved in THF unless otherwise noted, with a molecular concentration of $2.5-20 \mathrm{mM}$, and purged with argon. In some TRIR measurements, we observed baseline absorptions that amount to $\sim 10 \%$ of the peak absorptions. These baseline absorptions were subtracted from the reported spectra of the anions. The source of these absorptions may come from solvents or minor products of solutes after radiolysis.

Computations. Computations were carried out with Gaussian09. ${ }^{27}$ The geometries were optimized with the Hartree-Fock (HF) method and LC- $\omega \mathrm{PBE}^{28-30}$ or $\mathrm{B} 3 \mathrm{LYP}^{31,32}$ functional in density functional theory (DFT) calculations. The 6-31G(d) basis set was used unless otherwise noted. All calculations on anions were spin-unrestricted. All hexyl groups were replaced by ethyl groups. The geometry optimizations were performed without symmetry constraints unless otherwise noted. Frequency calculations were performed at the optimized geometries either with or without anharmonicity corrections. Reported frequencies are not scaled. Linear response timedependent DFT (TD-DFT) calculations were performed for low-lying excited states to determine transition energies at the ground state geometries. The atomic charges were derived from a leastsquares fit to the electrostatic potential calculated with the "Charges from electrostatic potentials using a Grid” (CHelpG) scheme ${ }^{33}$ as implemented in Gaussian09. A finite field method was used to determine changes in dipole moments between ground and TD-DFT excited states. ${ }^{34,35}$ Briefly, the excitation energy $\left(E_{\mathrm{op}}\right)$ in a static external electric field $(F)$ depends on the changes in dipole moment $\left(\Delta \mu_{12}\right)$ and polarizability $(\alpha)$ through the following equation 
$E_{o p}(F)=E_{o p}(0)-\Delta \mu_{12} F-\frac{1}{2} \Delta \alpha F^{2}$

where we applied electric fields of $F=0-0.002$ atomic units $(0-0.102 \mathrm{~V} / \AA)$ in the direction of the ground state's electronic dipole moment $\left(\mu_{1}\right)$ aligned along the long molecular axis. The electric dipole moment is defined relative to the center of nuclear charge to have non-zero values. We took into account the changes in the polarizability as well as in the dipole moment. Application of too strong a field results in changes in the orbitals involved in the transitions. The PCM model for solvation in THF was used, ${ }^{36-38}$ unless otherwise noted. Visualization was performed with GaussView 5.0.9.
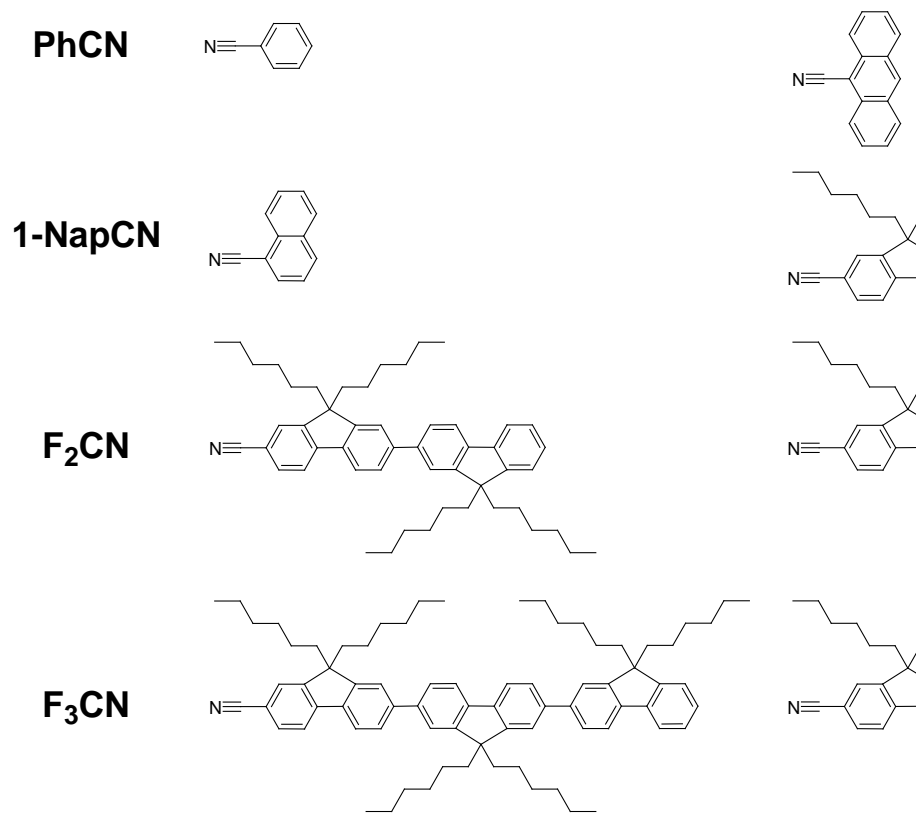

9-AntCN

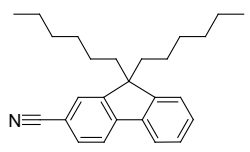

$\mathrm{F}_{1} \mathrm{CN}$
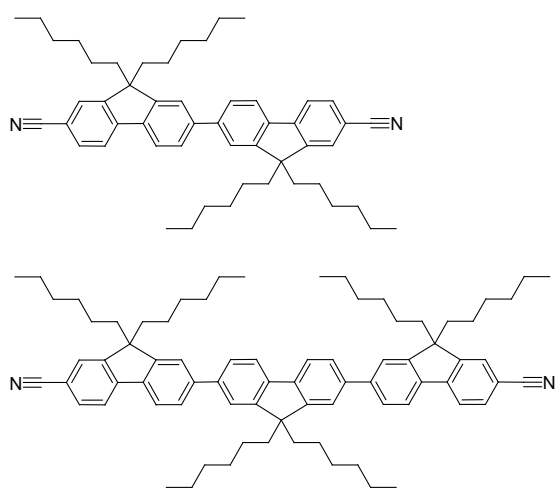

$\mathrm{F}_{3}(\mathrm{CN})_{2}$

Chart 1. The molecular structures of nitrile-functionalized aryl compounds used in this work. The abbreviated names are listed next to the molecules and are used throughout the paper. 


\section{Results}

\section{IR Shifts in Anion Radicals}

\subsection{Shift of Nitrile Vibration Upon reduction of mononitrile-functionalized aryl}

compounds (PhCN, 1-NapCN, 9-AntCN, $\mathbf{F}_{1} \mathbf{C N}, \mathbf{F}_{2} \mathbf{C N}$, and $\mathbf{F}_{3} \mathbf{C N}$, whose structures are shown in Chart 1 ), the $v(C \equiv N)$ IR bands shift to lower frequencies (Figure 1, Supporting Information Figure S2, and Table 1). Among PhCN and the fluorene oligomers, the difference between anion and neutral $(\Delta v)$ becomes smaller as the number of benzene rings increases. DFT calculations support the trend and predict that no more changes are expected going from $\mathbf{F}_{3} \mathbf{C N}$ to $\mathbf{F}_{4} \mathbf{C N}$. $\Delta v$ for 1-NapCN and 9-AntCN fall in between $\mathbf{F}_{\mathbf{1}} \mathbf{C N}$ and $\mathbf{F}_{\mathbf{2}} \mathbf{C N}$. Compared with the experimental values, the computed frequencies of both the neutral species and the anions are larger (Supporting Information Figure S3). In addition to harmonic frequency analysis, we calculated vibrational frequencies with anharmonicity corrections for $\mathbf{P h C N}$ and $\mathbf{F}_{\mathbf{1}} \mathbf{C N}$. Better agreements with the experimental data were observed with anharmonicity corrections, but $\Delta v$ did not improve.

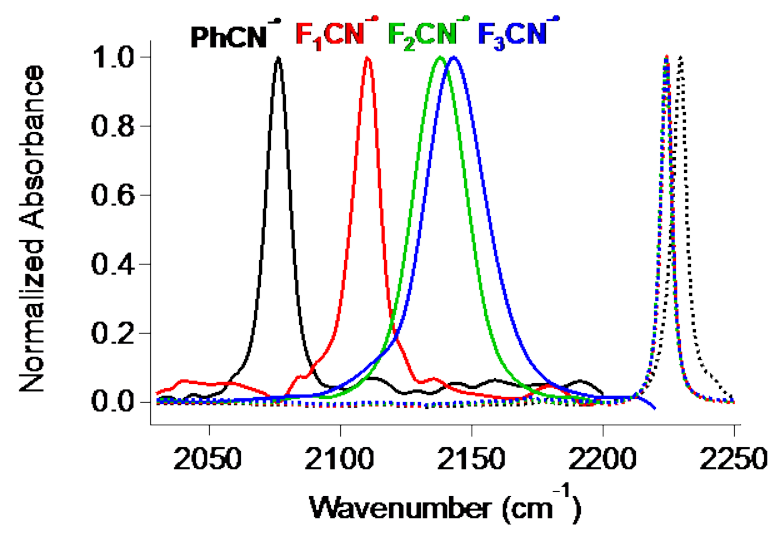

Figure 1. FTIR spectra of neutral molecules $\mathbf{P h C N}, \mathbf{F}_{\mathbf{1}} \mathbf{C N}, \mathbf{F}_{\mathbf{2}} \mathbf{C N}$, and $\mathbf{F}_{3} \mathbf{C N}$ in THF solution (dotted lines) and TRIR spectra of their free anions obtained 50 ns to $1 \mu$ s after pulse radiolysis (solid lines). All spectra are baseline subtracted and normalized. 
Table 1. Observed and Calculated $v(\mathrm{C} \equiv \mathrm{N})\left(\mathrm{cm}^{-1}\right)$ of Neutral and Anion Forms of Mononitrilefunctionalized Aryl Compounds in THF.

\begin{tabular}{|c|c|c|c|c|c|c|c|c|}
\hline \multirow{3}{*}{ Name } & \multicolumn{3}{|c|}{ Neutral } & \multicolumn{3}{|c|}{ Anion } & \multicolumn{2}{|c|}{$\Delta v^{a}$} \\
\hline & \multicolumn{2}{|c|}{ Expt } & \multirow{2}{*}{$\begin{array}{c}\omega \mathrm{PBE}^{b} \\
v_{\max }\end{array}$} & \multicolumn{2}{|c|}{ Expt } & \multirow{2}{*}{$\begin{array}{c}\omega \mathrm{PBE}^{b} \\
v_{\max }\end{array}$} & \multirow[b]{2}{*}{ Expt } & \multirow[b]{2}{*}{$\omega \mathrm{PBE}^{b}$} \\
\hline & $v_{\max }$ & $\begin{array}{c}\text { FWHM }^{c} \\
\left(\mathrm{~cm}^{-1}\right)\end{array}$ & & $v_{\max }$ & $\begin{array}{c}\text { FWHM }^{c} \\
\left(\mathrm{~cm}^{-1}\right)\end{array}$ & & & \\
\hline PhCN & 2230 & 6.48 & $\begin{array}{c}2308 \\
(2278)\end{array}$ & 2077 & 10.8 & $\begin{array}{c}2108 \\
(2067)\end{array}$ & -153 & $\begin{array}{l}-200 \\
(-211)\end{array}$ \\
\hline $\mathrm{F}_{1} \mathrm{CN}$ & 2224 & 4.97 & $\begin{array}{c}2301 \\
(2272)\end{array}$ & 2110 & 12.1 & $\begin{array}{c}2132 \\
(2103)\end{array}$ & -114 & $\begin{array}{c}-169 \\
(-169)\end{array}$ \\
\hline $\mathbf{F}_{2} \mathbf{C N}$ & 2224 & 4.93 & 2301 & 2138 & 24.1 & 2156 & -86 & -145 \\
\hline $\mathrm{F}_{3} \mathrm{CN}$ & 2224 & 5.11 & 2301 & 2143 & 27.0 & 2162 & -81 & -139 \\
\hline $\mathrm{F}_{4} \mathrm{CN}$ & - & - & 2301 & - & - & 2162 & - & -139 \\
\hline 1-NapCN & 2223 & 6.11 & 2299 & 2117 & 10.8 & 2140 & -106 & -159 \\
\hline 9-AntCN & 2216 & 5.56 & 2284 & 2138 & 5.21 & 2173 & -78 & -111 \\
\hline MeCN & - & - & $\begin{array}{c}2322 \\
(2279) \\
\end{array}$ & - & - & $\begin{array}{c}1718 \\
(1691) \\
\end{array}$ & - & $\begin{array}{c}-604 \\
(-588) \\
\end{array}$ \\
\hline
\end{tabular}

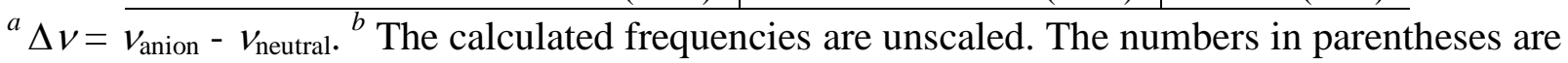
the calculated vibrations with anharmonicity corrections. All calculations used the functional LC- $\omega$ PBE $\left(\omega=0.1\right.$ bohr $\left.^{-1}\right)$ with the basis set $6-31 G(d)$ and the PCM model for solvation in THF except for MeCN that was calculated with 6-31G. ${ }^{c}$ Full width half maximum (FWHM) was determined by the fitting of the spectra by a single Voigt function, except for neutral PhCN which was fit by a Lorentzian.

1.2 Calibration Curve A linear correlation was obtained between the observed changes in $v(C$ $\equiv \mathrm{N})$ IR absorbance $(\Delta v)$ and the changes in the computationally determined charges on the CN groups from the neutral to the anion states ( $\Delta$ Charge): $\Delta$ Charge $=\left(q_{\mathrm{C}}+q_{\mathrm{N}}\right)_{\text {anion }}-$ $\left(q_{\mathrm{C}}+q_{\mathrm{N}}\right)_{\text {neutral }}$ except for the point 'Neutral' where $\Delta v$ and $\Delta$ Charge are set to 0 . A calibration curve was constructed (Figure 2), from which we can determine $\Delta$ Charge of the nitrile when $\Delta v$ is determined experimentally. A similar linear calibration curve was constructed with $\Delta$ Charge using a smaller basis set 3-21G (Supporting Information Figure S4). 


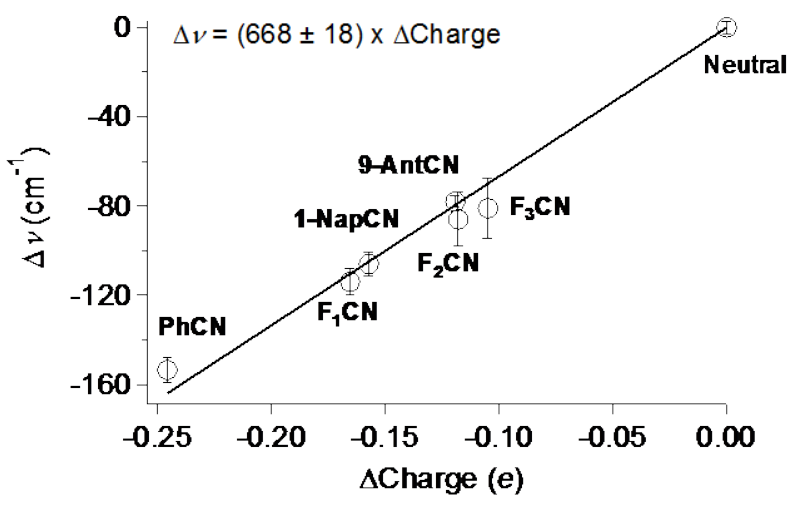

Figure 2. Calibration curve of the dependence of the experimentally observed changes in $\mathrm{v}(\mathrm{C} \equiv$ N) IR absorbance $(\Delta v)$ on the calculated changes in electrostatic potential CHelpG charges of the nitrile between the neutral and anion states ( $\Delta$ Charge). The bars represent the full width half maximum (FWHM) of the anion peaks. A linear fit was obtained $\left(\mathrm{R}^{2}=0.98\right)$.

According to the calibration curve, if one additional electron was placed solely on the nitrile ( $\Delta$ Charge $=-1.0$ ), hypothetically, we would expect a $-668 \mathrm{~cm}^{-1}$ difference from the neutral state. We obtained $\Delta v_{\text {comp }} \sim-600 \mathrm{~cm}^{-1}$ for acetonitrile, which can be considered as a hypothetical case (Table 1). A similar calibration curve can be constructed from $\Delta v_{\text {comp }}$, but its linearity is not as good (Supporting Information Figure S5). 
Table 2. Degree of Delocalization of Polarons of Nitrile-functionalized Aryl Compounds.

\begin{tabular}{|c|c|c|c|c|c|c|c|}
\hline \multirow[b]{2}{*}{ Molecules } & \multirow[b]{2}{*}{$\begin{array}{c}l_{\text {phys }}{ }^{c} \\
(\AA) \\
\end{array}$} & \multirow[b]{2}{*}{$\begin{array}{l}l_{\text {del }}^{d} \\
(\AA)\end{array}$} & \multicolumn{3}{|c|}{$\mathrm{ESP}^{a}$} & \multicolumn{2}{|c|}{$\mathrm{SOMO}^{b}$} \\
\hline & & & $\begin{array}{l}x^{(1)} \\
(\AA)\end{array}$ & $\begin{array}{c}\sigma \\
(\AA)\end{array}$ & $\gamma$ & $\begin{array}{l}x^{(1)} \\
(\AA)\end{array}$ & $\begin{array}{c}\sigma \\
(\AA)\end{array}$ \\
\hline PhCN & $\begin{array}{c}6.58 \\
(5.48)\end{array}$ & 6.58 & 0.10 & 2.07 & 0.28 & 0.19 & 1.97 \\
\hline $\mathrm{F}_{1} \mathrm{CN}$ & $\begin{array}{l}10.62 \\
(9.56)\end{array}$ & 10.62 & 0.78 & 3.14 & -0.08 & 0.97 & 2.86 \\
\hline $\mathrm{F}_{2} \mathrm{CN}$ & $\begin{array}{c}19.04 \\
(17.97)\end{array}$ & 19.04 & 2.77 & 4.87 & -0.52 & 3.07 & 4.23 \\
\hline $\mathbf{F}_{3} \mathbf{C N}$ & $\begin{array}{l}27.42 \\
(26.36)\end{array}$ & 22.10 & 6.24 & 5.75 & -0.84 & 6.66 & 4.81 \\
\hline $\mathrm{F}_{2} \mathrm{CN}_{2}$ & 20.54 & 20.54 & 0.00 & 5.87 & 0.00 & 0.00 & 5.32 \\
\hline $\mathrm{F}_{3} \mathrm{CN}_{2}$ & 28.96 & 22.10 & 5.90 & 6.33 & -0.88 & 6.31 & 5.40 \\
\hline $\begin{array}{c}\mathbf{F}_{\mathbf{3}} \mathbf{C N}_{\mathbf{2}} \\
\text { (C2 Symmetry) }\end{array}$ & 28.98 & - & 0.00 & 8.15 & 0.00 & 0.00 & 7.25 \\
\hline
\end{tabular}

${ }^{a}$ Calculated centroids $\left(x^{(1)}\right)$, halfwidths $(\sigma)$, and skewness $(\gamma)$ of anion excess charge, using ESP charge distributions, as described in Supporting Information Section II and eqs. S1-S5. ${ }^{b}$ Calculated centroids $\left(x^{(1)}\right)$, halfwidths $(\sigma)$, using the total density of anions SOMO. ${ }^{c} l_{\text {phys }}$ is the physical length of the molecule as an anion defined by the edge-to-edge distance between the nitrogen atom and the hydrogen or nitrogen atom at the opposite side of the molecule. The numbers in parentheses are the physical lengths of the molecule defined by the edge-to-edge distance between the nitrogen atom and the carbon atom at the opposite side of the molecule. ${ }^{d}$ $l_{\text {del }}=$ the edge-to-edge length of the anions defined based on IR information .

\subsection{Degree of Electron Delocalization}

We now estimate the degree of the spatial delocalization of the anions. Here, we limit our analysis to PhCN and the series of oligofluorenes as the changes in electron localization among the series are limited to one dimension. We can determine the edge-to-edge delocalization length $\left(l_{\text {del }}\right)$ based on $\Delta v(\mathrm{C} \equiv \mathrm{N})$. This method is similar in nature to the method based on redox potentials. ${ }^{5,6}$ DFT calculations show that an electron in the singly-occupied molecular orbital (SOMO) of the anionic state is delocalized over the entire molecule in $\mathbf{P h C N}, \mathbf{F}_{\mathbf{1}} \mathbf{C N}$, and $\mathbf{F}_{2} \mathbf{C N}$, while in all cases the nitrile group has more negative charge than other parts of the molecule. Figure S6 in Supporting Information shows that the experimentally determined $\Delta v$ has an inverse 
relationship with the physical length of the molecule for $\mathbf{P h C N}, \mathbf{F}_{\mathbf{1}} \mathbf{C N}$, and $\mathbf{F}_{\mathbf{2}} \mathbf{C N}$ : so $l_{\mathrm{del}}$ is equal to the physical length of these molecules (Table 2). On the other hand, computations predict that an electron in the SOMO of $\mathbf{F}_{3} \mathbf{C N}^{-}$does not span the entire molecule (vide infra). Based on the linear fit in Supporting Information Figure S6, we can estimate $l_{\text {del }}$ for $\mathbf{F}_{3} \mathbf{C N}^{-\bullet}$ as $22.1 \AA$, which is shorter than the physical length of $27.4 \AA$ (Table 2). The problem with these estimations of $l_{\text {del, }}$ or more generally with previously determined values of $l_{\text {del }}$, is that their physical definition is not clear. We consider them as the edge-to-edge length of polarons containing most ( 90\%) of the orbitals or charge densities, but the presence of a tail and asymmetric charge distribution prevents us from providing a concrete physical meaning.

\subsection{Widths of Polarons}

In an alternative definition, degrees of electron delocalization can be expressed as suitable second moments of the state charge densities, as discussed by Newton et al. ${ }^{39}$ Orbital variance has also been used as a means of determining localized molecular orbitals. ${ }^{40-42}$ It can be considered as the halfwidth of the polarons $(\sigma)$ and the width can be defined as $2 \sigma^{43}$ In order to distinguish $2 \sigma$ from the previously used $l_{\text {del }}$, we call them the width of polarons. We emphasize that both $l_{\text {del }}$ and $2 \sigma$ represent the degree of electron delocalization, but their definitions differ. We will seek to further clarify the relationships between experimentally measured $l_{\text {del }}$ and $2 \sigma$ in future studies. Following the treatments presented previously, ${ }^{39}$ we determined the width of the polarons based on the differences of ESP charge distributions between anions and neutrals as well as on the electron density in the SOMO of the anions (Table 2 and Supporting Information Table S1). A full density of the anion SOMO serves as a plausible zeroth-order estimate of the different densities of electrons to the extent that addition of an electron to the neutral does not 
appreciably polarize the 'core' defined by the occupied neutral orbitals. See Supporting Information Section II for details of the analysis. We obtained qualitatively similar values from

the two different analyses. The first moment shows that the centroid of the excess electron is shifted toward the nitrile. It is apparent that $\sigma<l_{\text {del }}$ and $\sigma$ increases from $\mathbf{P h C N}$ to $\mathbf{F}_{3} \mathbf{C N}$, showing that charges are more spatially spread out in the longer molecules. The skewness, $\gamma$, obtained from the third moment, confirms an asymmetric charge distribution for the mononitrilefunctionalized aryl compounds.

We would also like to note one interesting observation that the attachment of an electron at the ground state geometry of the neutral, which might be considered an "unrelaxed" electron, gives a slightly larger width and subsequent localization makes the width smaller (Supporting Information Table S1 and Figure S7). This resembles the observation that a "hot" exciton is more delocalized than a "thermalized" exciton. ${ }^{44}$

\section{Intramolecular Electron Localization and Exchange in Organic Mixed-Valence} Compounds

2.1 Two $\boldsymbol{v}(\mathrm{C} \equiv \mathrm{N})$ IR Peaks TRIR experiments producing the anions of $\mathbf{F}_{2}(\mathrm{CN})_{2}$ and $\mathbf{F}_{3}(\mathrm{CN})_{2}$ in THF (Chart 1) found, to our surprise, two $v(C \equiv N)$ IR absorptions (Figure 3a), along with the bleach of the neutral. FTIR spectra of the neutral species and an enlarged spectrum of $\mathbf{F}_{2}(\mathbf{C N})_{2}{ }^{-\bullet}$ are shown in Supporting Information Figure S8 and S9, respectively. 

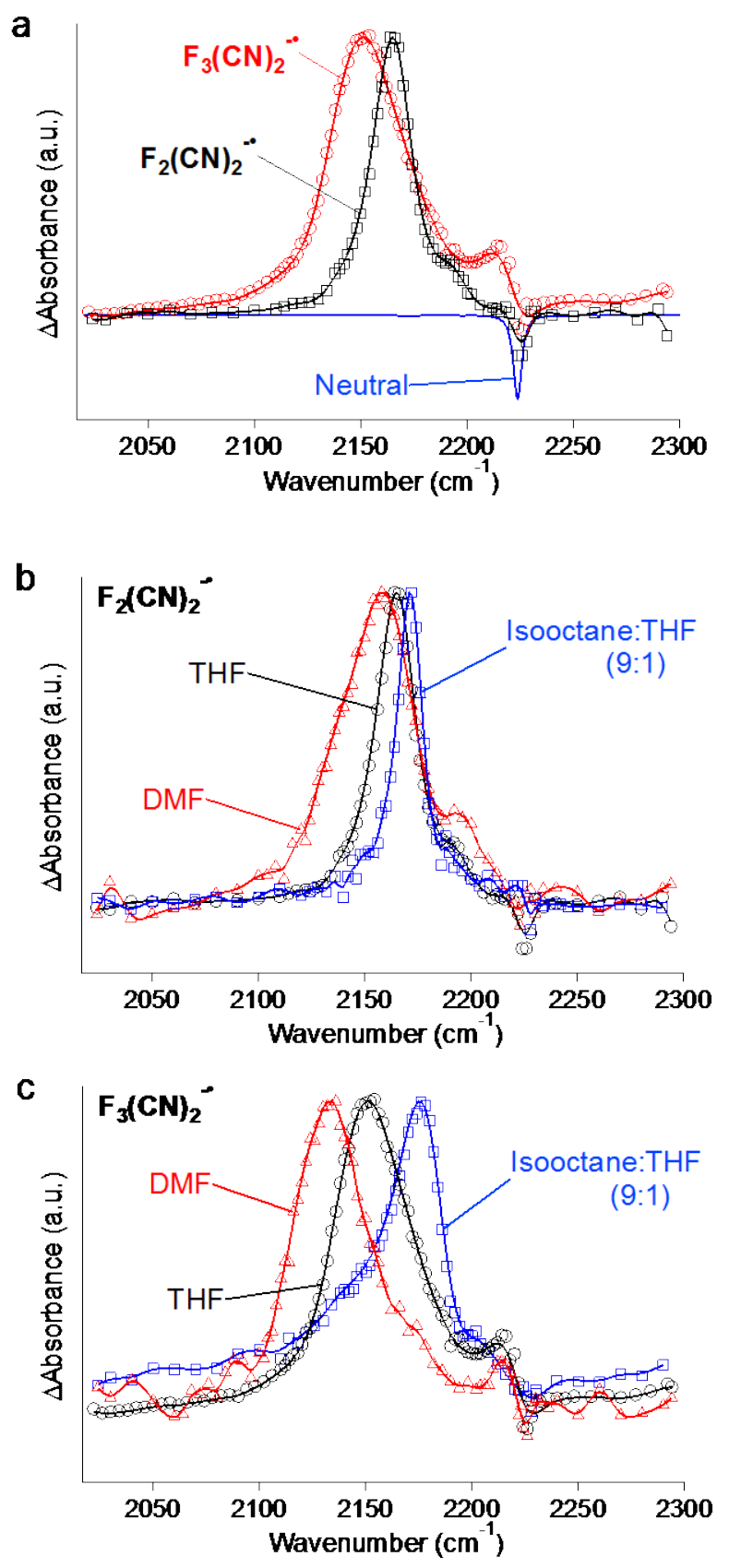

Figure 3. TRIR spectra of anions of $\mathbf{F}_{2}(\mathbf{C N})_{2}$ and $\mathbf{F}_{3}(\mathbf{C N})_{2}$ in the region of $v(\mathrm{C} \equiv \mathrm{N})$ obtained 40-50 ns after pulse radiolysis of the solutions. (a) In THF. The blue line is the inverted absorption spectrum of neutral $\mathbf{F}_{3}(\mathbf{C N})_{2}$. (b) $\mathbf{F}_{\mathbf{2}}(\mathbf{C N})_{2}{ }^{-\bullet}$ and (c) $\mathbf{F}_{3}(\mathbf{C N})_{2}{ }^{-\bullet}$ in isooctane:THF (9:1 by volume) (blue square), THF (black circle), and DMF (red triangle). The dips around 2220- 
$2230 \mathrm{~cm}^{-1}$ are the ground state bleaching of the neutrals. Typical peak absorbance ranges from 1-6 mOD.

Kaupp recently highlighted difficulties of computationally estimating electronic properties of mixed-valence compounds. ${ }^{45}$ Here, DFT calculations (Table 2) with the functional LC- $\omega$ PBE ( $\omega$ $=0.1$ bohr $^{-1}$ ) qualitatively predict electron localization in $\mathbf{F}_{3}(\mathbf{C N})_{2}{ }^{-}$while the functional B3LYP incorrectly predicts electron delocalization over the entire molecule. Self-interaction error, typical of standard functionals, causes an overdelocalization. ${ }^{46}$ LC- $\omega$ PBE was previously successfully used to reproduce exciton and polaron behaviors in oligofluorenes (particularly $\omega=$ 0.1 bohr $\left.^{-1}\right)^{6,47}$ as well as to capture important electronic parameters in organic mixed-valence radical anions of diquinones ${ }^{48}$ and bis(1,4-dimethoxybenzene) radical cations. ${ }^{14}$ When C2 symmetry is imposed, it predicts a single peak at $2170 \mathrm{~cm}^{-1}$ falling between the two peaks computed without symmetry. For $\mathbf{F}_{2}(\mathbf{C N})_{2}{ }^{-\bullet}$, on the other hand, both LC- $\omega$ PBE $(\omega=0.1)$ and B3LYP predict complete delocalization while a calculation with the HF method shows an extreme electron localization to one monomer unit (Supporting Information Figure S10). The optical results showing delocalization (Results Section 2.3 and Supporting Information Section IV) show the HF prediction to be incorrect. While increasing the value of $\omega$ in LC- $\omega \mathrm{PBE}(\omega>$ 0.2 bohr $^{-1}$ ) favors more complete localization and remains qualitatively consistent with IR results, use of $\omega=0.1$ bohr $^{-1}$ for $\mathbf{F}_{2}(\mathbf{C N})_{2}{ }^{-\bullet}$ is more consistent with the optical data.

To test if solvent-induced changes in localization affect the IR absorptions, we recorded the TRIR spectra in two other solvent systems. The $v(\mathrm{C} \equiv \mathrm{N})$ IR peaks are shifted to higher frequencies and become predominantly one peak in non-polar isooctane:THF (9:1 by volume), while they are shifted to lower frequencies and the presence of two peaks becomes more pronounced in polar $N, N$-dimethylformamide (DMF), especially in $\mathbf{F}_{3}(\mathbf{C N})_{2}{ }^{-}$(Figure $3 \mathrm{~b}$ and c). 
The changes here mainly stem from the changes in spatial delocalization of electrons within the molecules, i.e. a complete delocalization in isooctane:THF (9:1 by volume) and more localization in DMF, as supported by DFT calculations (Table 3). It is important to note that vibrational Stark effects (VSE) on the $v(\mathrm{C} \equiv \mathrm{N})$ bands through the changes in dipole moment of the solvents ${ }^{49,50}$ can also contribute to the shifts. Here, large contributions from changes in electron localization are almost certainly dominant, especially in $\mathbf{F}_{3}(\mathbf{C N})_{2}{ }^{-\bullet}$, but the contribution of the VSE will be explored in future studies.

Table 3. Observed and Calculated $v(\mathrm{C} \equiv \mathrm{N})\left(\mathrm{cm}^{-1}\right)$ of Neutral and Anion Forms of Dinitrilefunctionalized Oligofluorenes.

\begin{tabular}{|c|c|c|c|c|c|c|c|c|}
\hline \multirow{2}{*}{ Name } & \multicolumn{2}{|c|}{ Solvent } & \multicolumn{2}{|c|}{ Neutral } & \multicolumn{2}{|c|}{ Anion } & \multicolumn{2}{|c|}{$\Delta v^{g}$} \\
\hline & & $\begin{array}{c}\text { Dielectric } \\
\text { Constant } \\
\end{array}$ & $\operatorname{Expt}^{b}$ & $\omega \mathrm{PBE}^{c}$ & $\operatorname{Expt}^{b}$ & $\omega \mathrm{PBE}^{c}$ & $\operatorname{Expt}^{b}$ & $\omega \mathrm{PBE}^{c}$ \\
\hline \multirow{4}{*}{$\mathrm{F}_{2}(\mathrm{CN})_{2}$} & Vacuum & 1 & - & 2310 & - & 2244 & - & -66 \\
\hline & Isooctane & $\begin{array}{c}1.94 \\
(2.97)^{d}\end{array}$ & $\begin{array}{c}2227 \\
(4.47)\end{array}$ & 2306 & 2171 (15.5) & 2224 & -56 & -82 \\
\hline & THF & 7.52 & $\begin{array}{c}2224 \\
(4.73)\end{array}$ & 2301 & $\begin{array}{l}2165 \text { (23.0), } \\
2192 \text { (11.4) }\end{array}$ & 2187 & $-59,-32$ & -114 \\
\hline & DMF & 38.25 & $\begin{array}{c}2222 \\
(6.18) \\
\end{array}$ & 2299 & $\begin{array}{l}2158 \text { (43.9), } \\
2195 \text { (8.64) }\end{array}$ & 2177 & $-64,-27$ & -122 \\
\hline \multirow{4}{*}{$\mathrm{F}_{3}(\mathrm{CN})_{2}$} & Vacuum & 1 & - & 2309 & - & 2269 & - & -40 \\
\hline & Isooctane & $\begin{array}{c}1.94 \\
(2.50)^{d}\end{array}$ & $\begin{array}{l}2227 \\
(4.42)\end{array}$ & 2306 & 2175 (23.0) & 2264 & -52 & -42 \\
\hline & THF & 7.52 & $\begin{array}{c}2224 \\
(4.76)\end{array}$ & 2301 & $\begin{array}{l}2152 \text { (42.8), } \\
2213(27.5)\end{array}$ & 2167, 2298 & $-72,-11$ & $-134,-3$ \\
\hline & DMF & 38.25 & $\begin{array}{c}2222 \\
(6.18)\end{array}$ & 2299 & $\begin{array}{l}2134 \text { (43.2), } \\
2214 \text { (18.6) }\end{array}$ & 2153, 2298 & $-88,-8$ & $-146,-1$ \\
\hline \multicolumn{9}{|c|}{$\begin{array}{l}{ }^{a} \Delta v=v_{\text {anion }}-v_{\text {neutral. }}{ }^{b} \text { The numbers are the wavenumber }\left(\mathrm{cm}^{-1}\right) \text { at the maximum absorption. } \\
\text { The numbers in parentheses are FWHM determined by fitting with a Voigt function. }{ }^{c} \text { The } \\
\text { calculated frequencies are unscaled. All calculations used the standard basis set } 6-31 \mathrm{G}(\mathrm{d}) \text { and the } \\
\text { PCM model for solvation in isooctane, THF, or DMF. The functional LC- } \omega \text { PBE }\left(\omega=0.1 \text { bohr }^{-1}\right) \\
\text { was used. }{ }^{d} 10 \% \text { THF (by volume) in isooctane was used in the experiments for solubility } \\
\text { purposes. The values in parentheses are the calculated dielectric constant of } 10 \% \text { THF (by } \\
\text { volume) in isooctane assuming a simple linear relationship. }\end{array}$} \\
\hline
\end{tabular}


2.2 Reduction Potentials and Comproportionation Constants Reduction potentials for the nitrile-substituted fluorene compounds were determined by cyclic voltammetry in THF. The voltammograms are shown in Figure S11 and the values of the redox potentials are tabulated in Table 4 along with comproportionation constants, calculated by Eq 2.

$K_{\mathrm{c}}=\exp \left(\frac{F\left(E_{\mathrm{red}}^{\mathrm{n}+1}-E_{\mathrm{red}}^{\mathrm{n}}\right)}{R T}\right)=\exp \left(\frac{-F\left(\Delta G_{\mathrm{c}}^{\mathrm{n}}\right)}{R T}\right)$

where $\mathrm{n}$ is an integer, $F$ is the Faraday constant, $R$ is the molar gas constant, and $\Delta G_{\mathrm{c}}$ is expressed in units of eV. Details are in Supporting Information Section III.

Table 4. Reduction Potentials $\left(E_{\text {red }}, \mathrm{V} v s \mathrm{Fc}^{+/ 0}\right)^{a}$ and Comproportionation Constants $\left(K_{\mathrm{c}}\right)^{b}$

\begin{tabular}{cccccccc}
\hline Molecules & $1^{\text {st }} E_{\text {red }}$ & $K_{\mathrm{c}}{ }^{1}$ & $2^{\text {nd }} E_{\text {red }}$ & $\begin{array}{c}\Delta G_{\mathrm{c}}{ }^{1} \\
(\mathrm{eV})\end{array}$ & $K_{\mathrm{c}}{ }^{2}$ & $3^{\text {rd }} E_{\text {red }}$ & $\begin{array}{c}\Delta G_{\mathrm{c}}{ }^{2} \\
(\mathrm{eV})\end{array}$ \\
\hline $\mathbf{F}_{\mathbf{1}} \mathbf{C N}$ & $-2.72^{\mathrm{c}}$ & & & & & & \\
$\mathbf{F}_{\mathbf{2}} \mathbf{C N}$ & -2.60 & $7.53 \times 10^{6}$ & -3.00 & -0.40 & & & \\
$\left.\mathbf{F}_{\mathbf{2}} \mathbf{( C N}\right)_{2}$ & -2.45 & $9.00 \times 10^{3}$ & -2.68 & -0.23 & & & \\
$\mathbf{F}_{\mathbf{3}} \mathbf{C N}$ & -2.50 & $2.74 \times 10^{3}$ & -2.70 & -0.20 & $9.00 \times 10^{3}$ & -2.93 & -0.23 \\
$\mathbf{F}_{3}(\mathbf{C N})_{2}$ & -2.50 & 77.8 & -2.61 & -0.11 & $1.04 \times 10^{6}$ & -2.98 & -0.37
\end{tabular}

${ }^{a}$ Reduction potentials were determined in the presence of $0.1 \mathrm{M} \mathrm{TBA}^{+} \mathrm{PF}_{6}{ }^{-}$in THF. Uncertainties are $\pm 0.01-0.02 \mathrm{~V} .{ }^{b}$ Comproportionation constants $\left(K_{\mathrm{c}}\right)$ were evaluated by equation 2. ${ }^{c}$ A similar value of $-2.74 \mathrm{~V}$ was obtained with $0.1 \mathrm{M} \mathrm{TBA}^{+} \mathrm{BF}_{4}{ }^{-23}$ suggesting negligible effects from the anion of electrolyte.

\subsection{Electronic Transitions in Anions Chemical titrations with sodium biphenyl were} performed to obtain the absorption spectra of the reduced species (Figure 4). Anions, or negative polarons in conjugated molecules, exhibit two principal transitions in the NIR and visible regions, the $\mathrm{P}_{1}$ and $\mathrm{P}_{2}$ transitions, respectively. ${ }^{51}$ Here, the $\mathrm{P}_{1}$ and $\mathrm{P}_{2}$ transitions contain contributions mainly from the transitions from SOMO to SOMO+1 and from SOMO-1 to 
SOMO, respectively (orbitals are referred to those in the anions). The spectra of anions obtained chemically in the presence of Cryptand C222 show good agreement in the region of the $\mathrm{P}_{2}$ bands with those obtained radiolytically (Supporting Information Figure S12). However, the intensities of the $\mathrm{P}_{1}$ bands of the chemically reduced species, which are probably paired with $\mathrm{Na}^{+}$in the cryptand, are in some cases two times higher than those of the radiolytically produced species, which are free ions. These differences and the spectral linewidths of the optical transitions are discussed in Section IV of the SI.
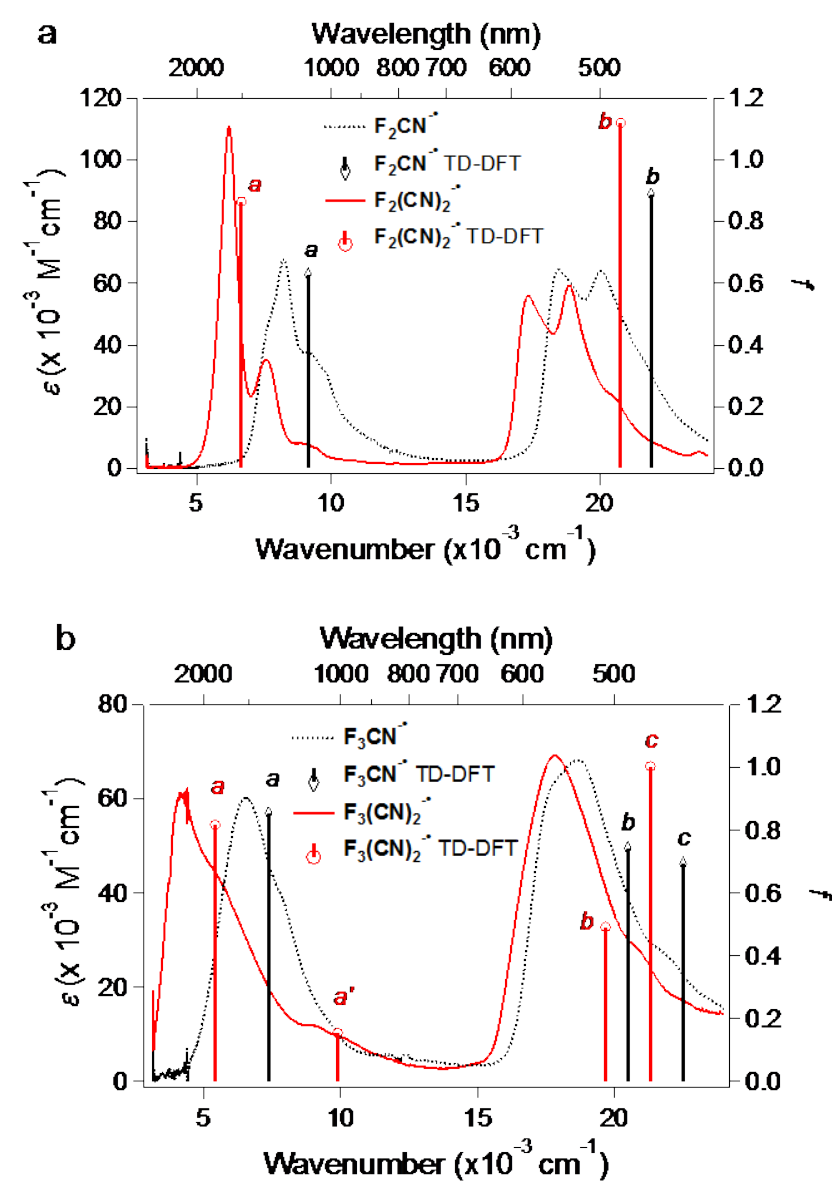

Figure 4. Comparison of experimental absorption spectra (left axis) of anions of (a) $\mathbf{F}_{2} \mathbf{C N}$ (black dotted) and $\mathbf{F}_{2}(\mathbf{C N})_{2}$ (red solid) and (b) $\mathbf{F}_{3} \mathbf{C N}$ (black dotted) and $\mathbf{F}_{3}(\mathbf{C N})_{2}$ (red solid) with the transitions calculated by TD-DFT (right axis). The absorption bands in the NIR region and the 
visible region are referred to as the $\mathrm{P}_{1}$ and $\mathrm{P}_{2}$ transitions, respectively. The transitions by TDDFT are labeled as $a$ and $a^{\prime}$ for the region of $\mathrm{P}_{1}$ transitions and $b$ and $c$ for the region of $\mathrm{P}_{2}$ transitions. Experimental spectra are recorded in THF and TD-DFT calculations are performed with a PCM model for solvation in THF. $\varepsilon$ is the molar absorption coefficient. $f$ is the oscillator strength.

The transition energies and oscillator frequencies calculated by TD-DFT/LC- $\omega$ PBE $(\omega=0.1)$ generally agree with the data in Figure 4 . The $P_{1}$ bands (the $a$ transition in Figure 4) mainly involve a transition from $\mathrm{SOMO}$ to $\mathrm{SOMO}+1$. Apart from $\mathbf{F}_{\mathbf{2}}(\mathbf{C N})_{2}{ }^{-\bullet}$, DFT calculations predict these transitions to have a charge-transfer character (Figure 5). This charge-transfer nature is reflected in the energies of the $\mathrm{P}_{1}$ transitions. While the $\mathrm{P}_{1}$ transition in $\mathbf{F}_{3}(\mathbf{C N})_{2}{ }^{-\bullet}$ transfers an electron between two similar orbitals, both with density on CN, the transition in $\mathbf{F}_{3}(\mathbf{C N})^{-\bullet}$ moves the electron to an orbital with little density on the $\mathrm{CN}$. The $\mathrm{P}_{1}$ transition in $\mathbf{F}_{3}(\mathbf{C N})^{-\bullet}$ has an energy higher roughly by the difference between redox potentials $E_{\text {red }}\left(\mathbf{F}_{3}(\mathbf{C N})\right)$ $E_{\text {red }}\left(\mathbf{F}_{3}\right)$. The computation predicts that there is another transition from the SOMO to SOMO+2 in $\mathbf{F}_{3}(\mathbf{C N})_{2}{ }^{-\bullet}$ (the $a^{\prime}$ transition in Figure $4 \mathrm{~b}$ ) in addition to the $\mathrm{P}_{1}$ band. An electron in $\mathrm{SOMO}+2$ is delocalized over the entire molecule. The absorption spectrum shows an unstructured small absorption corresponding to this predicted transition $a^{\prime}$. 


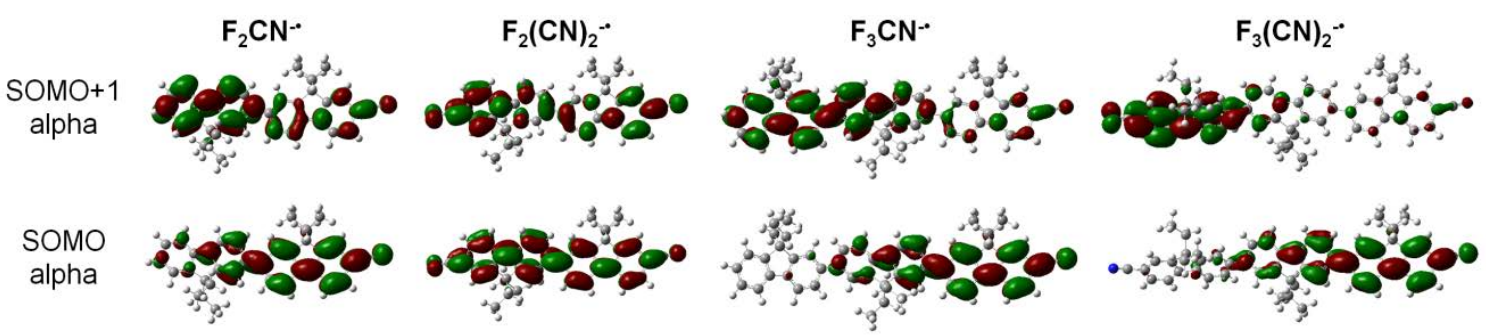

Figure 5. (a) Orbitals computed for $\mathbf{F}_{2} \mathbf{C N}^{-}, \mathbf{F}_{2}(\mathbf{C N})_{2}{ }^{-\bullet}, \mathbf{F}_{3} \mathbf{C N}^{-*}$, and $\mathbf{F}_{3}(\mathbf{C N})_{2}{ }^{-\bullet}$. All are computed by the LC- $\omega$ PBE functional $\left(\omega=0.1\right.$ bohr $\left.^{-1}\right)$, using the $6-31 \mathrm{G}(\mathrm{d})$ basis set and the PCM model for solvation in THF. All surfaces have an isovalue of 0.02 . Note that the calculation predicts the complete delocalization of the electron in $\mathbf{F}_{2}(\mathbf{C N})_{2}{ }^{-}$.

\subsection{Calculations of Electronic Couplings}

I. GMH Theory We have extracted diabatic electronic couplings, $H_{\mathrm{ab}}$, and other electron transfer parameters from the available computational and experimental data. An important approach to calculate the electronic coupling is the generalized Mulliken-Hush (GMH) theory. ${ }^{52,53}$ In the framework of parabolic diabatic states of the initial and final electronic states, the observed band maximum for a charge transfer transition, $E_{\mathrm{op}}$, is equal to the total vertical energy $\left(\lambda+\Delta G^{0}\right)$. Since the free energy change $\Delta G^{0}$ is equal to 0 for a symmetrical electron transfer in $\mathbf{F}_{2}(\mathbf{C N})_{2}{ }^{-\bullet}$ and $\mathbf{F}_{3}(\mathbf{C N})_{2}{ }^{-\bullet} E_{\text {op }}$ should equal $\lambda .^{54}$ Hush related $H_{\mathrm{ab}}$ to $E_{\text {op }}$ upon electron transfer through equation 3 ,

$H_{\mathrm{ab}}=\frac{\mu_{12} E_{\mathrm{op}}}{\Delta \mu_{\mathrm{ab}}}$

where a and b represent ground (initial) and excited (final) states of the system on diabatic surfaces, $\mu_{12}$ is the transition dipole moment coupling the adiabatic surfaces of the ground and excited states, and $\Delta \mu_{\mathrm{ab}}$ is the change in dipole moment between the ground and excited states. 
Newton and Cave ${ }^{52,53}$ extended this theory by showing that $\Delta \mu_{\mathrm{ab}}$ can be written in terms of the experimentally accessible adiabatic parameters $\Delta \mu_{12}$, the change in dipole moment between the ground $\left(\mu_{1}\right)$ and excited $\left(\mu_{2}\right)$ states where 1 and 2 represent each state on adiabatic surfaces. Now, $H_{\mathrm{ab}}$ is calculated by equation 4 .

$H_{\mathrm{ab}}=\frac{\mu_{12} E_{\mathrm{op}}}{\left(\left(\Delta \mu_{12}\right)^{2}+4\left(\mu_{12}\right)^{2}\right)^{1 / 2}}$

$\Delta \mu_{\mathrm{ab}}$ and $\Delta \mu_{12}$ are related to the diabatic and adiabatic electron transfer distance through $d_{\mathrm{ab}} \equiv$ $\Delta \mu_{\mathrm{ab}} / e$ and $d_{12} \equiv \Delta \mu_{12} / e$. Experimental measures by techniques such as Stark spectroscopy and electron spin resonance (ESR) spectroscopy yield adiabatic distances, $d_{12}$, which are shorter than diabatic distances, $d_{\mathrm{ab}}{ }^{55}$ The beauty and popularity of the GMH analysis rests on the fact that we do not have to explicitly define diabatic states, an often difficult task. Subotnik et al. further generalized GMH to multiple and nonlinear charge centers through the Boys localization method. $^{56}$

In Table 5 values of $H_{\mathrm{ab}}$ and other parameters determined by GMH are listed along with comparison to estimates from other methods. The first two methods are based on semi-empirical GMH theory, in which all the parameters are derived from photophysical measurements except for $\Delta \mu_{12}$. Experimental determination of $\Delta \mu_{12}$ is one of the difficulties of using GMH analysis. ${ }^{22}$ Experimentally, $\Delta \mu_{12}$ can be determined by Stark spectroscopy. ${ }^{57}$ Here, we instead employed a finite field method to computationally estimate $\Delta \mu_{12}$ for GMH analysis ${ }^{34,35}$ using equation 1 . An excellent fit to the quadratic equation was obtained when changes in the polarizability were taken into account (Supporting Information Figure S13). We have evaluated $\mu_{12}$ by two methods. One is by the Hush approximation ${ }^{54}$ (GMH Opt 1)

$\mu_{12}($ Hush $)=n_{\text {cor }} 0.09854\left(\varepsilon_{\max } \Delta v_{1 / 2} / E_{\text {op }}\right)^{1 / 2}$ 
where $\varepsilon_{\max }$ is the extinction coefficient at the maximum wavenumber. The other (GMH Opt 2) integrates the absorption band including the vibronic structure, and is sometimes referred to as Liptay’s formulation. $^{58}$.

$\mu_{12}($ Liptay $)=n_{\text {cor }} 0.09854\left(\int_{\text {band }} \frac{\varepsilon(\bar{v})}{\bar{v}} \mathrm{~d} \bar{v}\right)^{1 / 2}$

In both cases, the solvent refractive index correction $\left(n_{\text {cor }}\right)$ was applied: $n_{\text {cor }}=\frac{3 \sqrt{n}}{\left(n^{2}+2\right)}$ where $\mathrm{n}$ is solvent refractive index. ${ }^{59,60}$ The two methods give comparable results. The third method is still within the framework of GMH, but we determined the adiabatic electron transfer distance, $d_{12}$ using our new IR-CHARGE method described in the following paragraph, instead of using optically-derived values. Importantly, this method does not rely on the measurement of $\Delta \mu_{12}$ by Stark spectroscopy or computational methods. The IR-CHARGE method is semi-empirical in nature. In addition, the moment analysis on the ESP charge distributions (Table 2) provides another estimate of $d_{12}=2 x^{(1)}=11.8 \AA$ (by ESP charge-based method) and $12.6 \AA$ (by SOMObased method) for $\mathbf{F}_{3}(\mathbf{C N})_{2}{ }^{-\bullet}$, which are $\sim 1-2 \AA$ larger, but still close to those obtained by GMH analysis. We would also like to note that ion pairing appears to increase the transition dipole moment $\mu_{12}$, especially for $\mathbf{F}_{\mathbf{2}}(\mathbf{C N})_{2}^{-\bullet}$ (vide supra). We therefore report $H_{\mathrm{ab}}$ and other parameters for $\mathbf{F}_{\mathbf{2}}(\mathbf{C N})_{2}^{-\bullet}$ based on the radiolyitcally determined spectrum, the free ions in which have an extinction coefficient $\sim 0.45$ times that of the chemically-obtained spectrum. To compare with $\mathbf{F}_{3}(\mathbf{C N})_{2}$, we also used the value determined from the chemically produced spectrum. Lastly, we make a computational estimate of $H_{\mathrm{ab}}$ using Koopman's theorem (KT). Here, we obtain a transition state as a C2 symmetrized structure by DFT calculations. Then $H_{\mathrm{ab}}$ is estimated as half the difference in energies of the LUMO and LUMO+1 orbitals of the neutral. 
Table 5. Electronic Coupling Analysis ${ }^{a}$

\begin{tabular}{|c|c|c|c|c|c|c|c|c|c|c|}
\hline \multirow{2}{*}{ Molecules } & \multirow{2}{*}{ Methods } & $E_{\mathrm{op}}=\lambda$ & $\Delta \mu_{12}$ & $d_{\mathrm{NN}}^{b}$ & $d_{12}{ }^{c}$ & $\mu_{12}$ & $\Delta \mu_{\mathrm{ab}}$ & $d_{\mathrm{ab}}^{c}$ & $H_{\mathrm{ab}}{ }^{d}$ & $\Delta G^{\ddagger}$ \\
\hline & & $\mathrm{eV}$ & $\mathrm{D}$ & $\AA$ & $\AA$ & $\mathrm{D}$ & $\mathrm{D}$ & $\AA$ & $\mathrm{eV}$ & $\mathrm{eV}$ \\
\hline \multirow{6}{*}{$\mathrm{F}_{3}(\mathrm{CN})_{2}$} & GMH Cal & 0.671 & -51.88 & 28.96 & 10.8 & 17.9 & 63.1 & 13.1 & 0.190 & 0.031 \\
\hline & $\begin{array}{c}\text { GMH Cal } \\
\text { C2 Symmetry }\end{array}$ & 0.437 & & 28.98 & & & & & 0.220 & $0.039^{e}$ \\
\hline & GMH Opt 1 & 0.506 & -51.88 & 28.96 & 10.8 & 18.4 & 67.1 & 14.0 & 0.140 & 0.026 \\
\hline & GMH Opt 2 & 0.506 & -51.88 & 28.96 & 10.8 & 18.9 & 64.2 & 14.1 & 0.150 & 0.021 \\
\hline & $\begin{array}{l}\text { GMH IR- } \\
\text { CHARGE }\end{array}$ & 0.506 & -- & 28.96 & 10.6 & 18.9 & 67.2 & 14.0 & 0.140 & 0.024 \\
\hline & $\begin{array}{c}\text { KT } \\
\text { C2 Symmetry } \\
\end{array}$ & & & 28.90 & & & & & 0.140 & \\
\hline \multirow{4}{*}{$\mathrm{F}_{2}(\mathrm{CN})_{2}$} & $\mathrm{GMH} \mathrm{Cal}^{f}$ & 0.825 & & 20.54 & & & & & 0.410 & 0 \\
\hline & $\mathrm{GMH} \mathrm{Opt}^{g}$ & 0.767 & & 20.54 & & & & & 0.380 & 0 \\
\hline & $\begin{array}{l}\text { GMH IR- } \\
\text { CHARGE }^{h}\end{array}$ & 0.767 & -- & 20.54 & 3.1 & $\begin{array}{l}12.8^{i} \\
(8.6)\end{array}$ & $\begin{array}{c}32.9 \\
(24.6)\end{array}$ & $\begin{array}{c}6.9 \\
(5.1)\end{array}$ & $\begin{array}{c}0.300 \\
(0.270)\end{array}$ & $\begin{array}{c}0.010 \\
(0.018)\end{array}$ \\
\hline & $\begin{array}{c}\text { KT } \\
\text { C2 Symmetry }\end{array}$ & & & 20.42 & & & & & 0.310 & \\
\hline
\end{tabular}

${ }^{a}$ All the experimental values are determined in THF. All calculations used the standard basis set 6-31G(d) and the PCM model for solvation in THF. The functional LC- $\omega$ PBE $\left(\omega=0.1 \mathrm{bohr}^{-}\right.$ ${ }^{1}$ ) was used. ${ }^{b} d_{\mathrm{NN}}$ is the edge-to-edge (N-to-N) distance of the molecules determined at the optimized structures of the anions except for those listed in KT C2 Symmetry that are the distances of the neutrals. ${ }^{c}$ Uncertainties are $\pm 0.2 \AA \AA{ }^{d}$ Uncertainties are $\pm 0.01 \mathrm{eV} .{ }^{e} \Delta G^{\ddagger}=E_{0}(\mathrm{C} 2$ Symmetry) - $E_{0}$ (localized). ${ }^{f}$ DFT calculation of $\quad \mathbf{F}_{\mathbf{2}}(\mathbf{C N})_{\mathbf{2}}{ }^{\bullet}$ predicts a complete delocalization and thus $H_{\mathrm{ab}}=E_{\mathrm{op}} / 2 .{ }^{g}$ Assumed a complete delocalization of the electron. ${ }^{h}$ The numbers in parentheses are determined by using $\mu_{12}$ determined from the radiolytically-produced spectrum. ${ }^{i}$ $\mu_{12}$ based on Liptay’s formulation with solvent correction (Eq. 6).

II. The IR-CHARGE Method $\quad H_{\mathrm{ab}}$ can be obtained using adiabatic electron transfer distances, $d_{12}$, derived from the IR spectra. Here, we call this the IR-CHARGE method. Briefly, we use the nitrile vibration as a convenient reporter for charge distribution within the molecule. A detailed explanation is given in Supporting Information Section V. The obtained $d_{12}$ for $\mathbf{F}_{3}(\mathbf{C N})_{2}{ }^{-\bullet}$ is very similar to those based on the computed value (Table 5). This comparison is encouraging, although by no means do we intend for it to stand as proof of the validity of our 
method. It is important to note that the second of two assumptions (see Supporting Information) is necessary to calculate the center of charge and any changes in this assumption can have a sizable impact on the determination of $d_{12}$. Nonetheless, at present, this IR-CHARGE method allows us to estimate $\Delta \mu_{12}$ from which we can experimentally determine the coupling strength, $H_{\mathrm{ab}}$, and the activation energy for electron transfer in $\mathbf{F}_{\mathbf{2}}(\mathbf{C N})_{2}{ }^{-\bullet}$ that exhibits both localization and delocalization behaviors. It may be interesting to see if we can obtain similar results by Stark

spectroscopy. ${ }^{57} H_{\mathrm{ab}}$ was calculated based on equation 4 , using the experimentally available parameters of $\mu_{12}$ and $E_{\mathrm{op}}$. The $H_{\mathrm{ab}}$ value for $\mathbf{F}_{2}(\mathbf{C N})_{2}{ }^{-\bullet}$ obtained based on IR-CHARGE is $\sim 80$ $100 \mathrm{meV}$ smaller than the values from the computational and optical GMH methods that assume a complete delocalization of the electron, and it is close to the value calculated by KT.

\section{Discussion}

\section{IR Shifts in Anion Radicals}

1.1 Structural Changes and Excess Electron Junchnovski and Binev reported early examples $^{61,62}$ of large $v(C \equiv N)$ IR shifts upon chemical reduction of nitrile-functionalized arenes. While their spectral analysis was complicated due to the presence of ion pairing with metal ions or electrolytes, they concluded that their large IR shifts stem from "the very great mobility of the additional electron occupying the antibonding orbital.”61 This picture still holds for our case. For the neutral molecules in Chart $1, v(C \equiv N)$ changes little. In clear contrast, the $v(C \equiv N)$ IR band exhibits dramatic shifts when the molecules are reduced to anions. The degree of shifting is clearly dependent upon the degree of electron localization; from $-153 \mathrm{~cm}^{-1}$ for the shortest PhCN ${ }^{-\bullet}$ to $-81 \mathrm{~cm}^{-1}$ for the longest $\mathbf{F}_{3} \mathbf{C N}^{-*}$. The linewidths (FWHM) of the IR spectra, given in Table 1, are all near $5 \mathrm{~cm}^{-1}$ for the neutrals. The linewidth is similar, $5.2 \mathrm{~cm}^{-1}$, for $\mathbf{9}-\mathbf{A n t C N}^{-}$, but 
increases to $10.8 \mathrm{~cm}^{-1}$ for $\mathbf{P h C N} \mathbf{C N}^{-}$, and further to $27.0 \mathrm{~cm}^{-1}$ for $\mathbf{F}_{3} \mathbf{C N}^{-*}$. In contrast, the changes in FWHM of the $v(\mathrm{C} \equiv \mathrm{N})$ IR band in the neutral species are very small, mainly coming from solvent interactions. The large increases in the line broadening in the anions of oligofluorenes can be understood as fluctuations in the charges on the nitrile due to structural fluctuations, particularly those driven by fluctuations of dihedral angles associated with single C-C bonds.

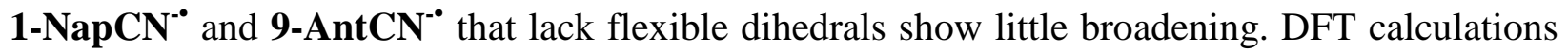
give insight. For $\mathbf{F}_{3} \mathbf{C \mathbf { N } ^ { * }}$ the calculations predict $v(\mathrm{C} \equiv \mathrm{N})$ shifts to lower energy when the dihedral angles are fixed to favor electron localization to the nitrile end (perpendicular conformation, Supporting Information Figure S14a) and to higher energy when they are fixed to favor electron delocalization (planar conformation, Supporting Information Figure S14b). A perpendicular conformation partly divides the $\mathbf{F}_{3} \mathbf{C N}^{-}$into $\mathbf{F}_{\mathbf{1}} \mathbf{C} \mathbf{N}^{-}$and $\mathbf{F}_{2}$ moieties whose reduction potentials are within a couple of $k_{\mathrm{B}} T{ }^{6}$ The small energy difference allows an electron to move from the $\mathbf{F}_{1} \mathbf{C N}^{*}$ side to the $\mathbf{F}_{2}$ side, leaving the $\mathrm{CN}$ group much less negative. While such a perpendicular conformation is an extreme case, ${ }^{47}$ we illustrate that the observed changes in peak shifts and linewidths of the $v(\mathrm{C} \equiv \mathrm{N})$ IR absorptions show a clear structural change accompanying changes in electronic density distribution.

\subsection{Calibration Curve Previous studies that examined the use of specific IR frequencies} to probe electron transfer reactions focused on carbonyl frequencies; first studied by Volk et al. on ferrocenophanone ${ }^{10}$ and later by Rubtsov et al. on Zinc porphyrins ${ }^{11}$ and then by Kim et al. on Ruthenium complexes. ${ }^{63}$ A related recent study by Koch et al. ${ }^{15}$ showed that nitrile frequencies can be used to distinguish between charge-transfer states and local excited states using TRIR. The calibration curve constructed here establishes a quantitative connection between the 
observed spectral shifts and changes in charge distribution. While the particular calibration curve constructed here should be used only in THF, similar calibration curves can be constructed for other solvents; such studies are currently underway. The general approach presented here is useful to estimate electronic density distributions in molecules with appropriate IR reporters. Such examples may include studies of the long-standing problem of charge-transfer states in 4(dimethylamino)benzonitrile (DMABN) and other similar compounds. ${ }^{64}$

\section{Intramolecular Electron Localization and Exchange in Organic Mixed-Valence}

\section{Compounds}

2.1 Degree of Electron Localization The reduction potentials (Table 4) of $\mathbf{F}_{3} \mathbf{C N}$ and $\mathbf{F}_{3}(\mathbf{C N})_{2}$ are the same indicating that the electron is not delocalized over both nitrile groups, while the potentials show that $\mathbf{F}_{\mathbf{2}}(\mathbf{C N})_{2}{ }^{-\bullet}$ is stabilized $150 \mathrm{mV}$ more than $\mathbf{F}_{\mathbf{2}} \mathbf{C N}^{-\bullet}$ supporting partial delocalization over both nitriles in $\mathbf{F}_{\mathbf{2}}(\mathbf{C N})_{2}{ }^{-\bullet}$. Thus, redox potentials corroborate findings based on the IR results. Additional support comes from optical spectra such as the narrow bandwidth of the $\mathrm{P}_{1}$ band in $\mathbf{F}_{\mathbf{2}}(\mathbf{C N})_{2}{ }^{-\bullet}$, as described in Supporting Information Section VI.

\subsection{Origin of Two $\boldsymbol{v}(\mathbf{C} \equiv \mathbf{N})$ IR Peaks The observation of two distinct $v(C \equiv N)$ IR peaks} in the anions of dinitrile-substituted oligofluorenes points to partial or complete localization. Application of IR spectroscopy to organic mixed-valence compounds was first reported by Mazur and co-workers in their studies of dicarbonyl radical anions. ${ }^{65,66}$ Kubiak and Ito have further applied IR to inorganic mixed-valence complexes in the form of IR spectroelectrochemistry. ${ }^{67,68}$ In their studies of inorganic mixed-valence compounds, they identified localization and delocalization of an exchanging electron by the coalescence of 
$v(\mathrm{C}=\mathrm{O})$ bands at metal redox centers. Hoekstra et al. previously observed two Raman peaks for the central $v(\mathrm{C} \equiv \mathrm{C})$ in 4,4'-dinitrolane radical anion, which they assigned to the localized and delocalized species in equlibrium. ${ }^{69}$ The data in Figure 3 find that larger solvent polarity favors localization as does the longer distance between nitriles in $\mathbf{F}_{3}(\mathbf{C N})_{2}{ }^{-}$. The width $(2 \sigma)$ of the polaron (Table 2) is large enough, enabling it to physically delocalize over the two "redox" centers, albeit asymmetrically, which contrasts with typical inorganic and some organic mixedvalence compounds where the widths of the polaron are smaller. These observations, including the slight, apparent indication of some localization in $\mathbf{F}_{\mathbf{2}}(\mathbf{C N})_{2}{ }^{-\bullet}$ are discussed in Section VII of the Supporting Information.

2.3 Electron Transfer and Couplings The electron transfer distances obtained above using the IR-CHARGE method are much smaller than the edge-to-edge distances, $d_{\mathrm{NN}}$. From IR-CHARGE, the electron moves over $3.1 \AA$ on adiabatic surfaces when an electron transfers in $\mathbf{F}_{\mathbf{2}}(\mathbf{C N})_{2}{ }^{-\bullet}$ and $\sim 10-11 \AA$ in $\mathbf{F}_{3}(\mathbf{C N})_{2}{ }^{-\bullet}$. Electron transfer distances are expected to be smaller than the edge-toedge distance in organic mixed-valence compounds because the electron is delocalized over many atoms, unlike in inorganic mixed-valence compounds in which charges are concentrated on the metals. When an electron moves from one side to the other in a symmetric molecule like $\mathbf{F}_{3}(\mathbf{C N})_{2}{ }^{-\bullet}$, the transition state (TS) is expected to have C2 symmetry. We optimized the geometry of $\mathbf{F}_{3}(\mathbf{C N})_{2}{ }^{-\bullet}$ with a C2 symmetry constraint and assigned this as a TS. The energy computed at this geometry is $\sim 39 \mathrm{meV}$ higher than the computed energy without symmetry, in close agreement with the energy barrier estimated by the GMH method based on entirely computational values (vide infra). With C2 symmetry the electron is not well delocalized over the two nitriles, but resides principally in the fluorenes between them (Supporting Information 
Figure S15). Still the $v(\mathrm{C} \equiv N)$ IR is shifted to lower frequency compared to that in the neutral $\left(\Delta v_{\text {comp }}=-131 \mathrm{~cm}^{-1}\right.$, see Results Section 2.1). In addition, the electronic couplings determined for a localized state (190 meV from the calculation only) and a TS state (220 meV) are comparable, showing that the electronic coupling is independent of the system's coordinates, namely the Condon approximation.

The electronic coupling in $\mathbf{F}_{2}(\mathbf{C N})_{2}{ }^{-{ }^{-}}$is about twice that in $\mathbf{F}_{3}(\mathbf{C N})_{2}{ }^{-\bullet}$, showing a stronger interaction between the two redox "centers". The coupling in $\mathbf{F}_{2}(\mathbf{C N})_{2}^{-\bullet}$ estimated by the IRCHARGE method may be considered to be a lower limit while that obtained by the GMH $\mathrm{Cal} / \mathrm{Opt}$ and KT can be upper limits, so the actual coupling may lie between these two close estimates. In the IR-CHARGE method, the electron is assumed to be transiently localized over a short distance $\left(d_{12}=3.1 \AA\right)$ on the IR timescale, still producing sizable changes in dipole moments when it moves.

The coupling values determined by both computation and experiments are purely electronic. It is expected that if we take into account Franck-Condon factors, ${ }^{70}$ then we may have slightly smaller values. Therefore, the values reported here are considered upper limits. While we did not compute diabatic states specifically, it would be interesting to see if we can recover the diabatic electron transfer distances from computations.

2.4 Small Energy Barriers Creutz and Taube stated decades ago ${ }^{17,18}$ that the importance of mixed-valence compounds is the possibility of calculating the activation energy $\left(\Delta G^{\ddagger}\right)$ associated with electron transfer. In the current system, we do not have a clear distinction between redox centers and bridges unlike in many inorganic mixed-valence compounds and some other organic counterparts. Nevertheless, the GMH analysis using the optical or IR-CHARGE approach allows 
us to calculate the necessary parameters to obtain the activation energies in our systems. The activation energies (reported in Table 5) are calculated from the following equation, ${ }^{71}$

$\Delta G^{\ddagger}=\frac{\left(E_{\mathrm{op}}-2 H_{\mathrm{ab}}\right)^{2}}{4 E_{\mathrm{op}}}$

which depends on both the reorganization energy and the electronic coupling. As the electronic coupling is not negligible compared to the reorganization energy, the activation energies become lower than the classical Marcus limit of $\lambda / 4$ for a non-adiabatic electron transfer. The obtained $\Delta G^{\ddagger}$ are $\sim 10 \mathrm{meV}$ for $\mathbf{F}_{\mathbf{2}}(\mathbf{C N})_{2}{ }^{-\bullet}$ and $\sim 20 \mathrm{meV}$ for $\mathbf{F}_{3}(\mathbf{C N})_{2}{ }^{-}, \sim 1 / 2$ to $1 k_{\mathrm{B}} T$ at room temperature $\left(k_{\mathrm{B}} T \sim 25 \mathrm{meV}\right.$ at $\left.293 \mathrm{~K}\right)$. A pictorial adiabatic potential energy surface for $\mathbf{F}_{3}(\mathbf{C N})_{2}{ }^{-{ }^{*}}$ is shown in Figure 6. We may have slightly different values if we take into account the Franck-Condon factors in the electronic coupling, but changes are expected to be small.

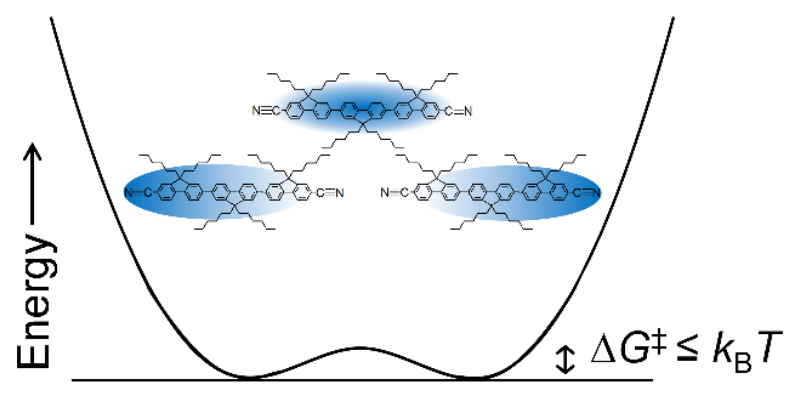

ET Coordinate $\rightarrow$

Figure 6. Pictorial representation of the adiabatic potential energy surface together with electron localization within $\mathbf{F}_{3}(\mathbf{C N})_{2}{ }^{-\bullet}$.

These values are at least four times smaller than reported $\Delta G^{\ddagger}$ for hole transfers in similar systems $^{14,60,72-76}$ Our values are closer to the value for hole transfer in the smallest triarylaminebased organic mixed-valence compounds studied by Lambert and Nöll; where $\Delta G^{\ddagger}$ of $\sim 29 \mathrm{meV}$ 
is reported for $\mathrm{N}, \mathrm{N} \mathrm{N}^{\prime}, \mathrm{N}^{\prime}$-tetra-4-methoxyphenyl-p-phenylenediamine in DCM, whose triarylamine redox center-to-center distance is only $5 \AA \AA^{77,78}$ As the redox sites are well coupled to the bridges in our systems, we can exclude factors arising from the connection between the redox centers and the bridges as a possible source of activation energy. Thus, we reason that the source of these small activation energies originates from the movements of dihedral angles connecting the fluorene units and from solvent reorganization. It is known that oligo- and polyfluorenes become flatter (smaller dihedral angles) when an electron is added. ${ }^{6,79}$ As the electron is already delocalized ${ }^{80}$ in $\mathbf{F}_{\mathbf{2}}(\mathbf{C N})_{2}{ }^{-*}$, the necessary changes associated with an electron shift are expected to be smaller than those in $\mathbf{F}_{3}(\mathbf{C N})_{2}{ }^{-\bullet}$ where the electron is only partially delocalized. In $\mathbf{F}_{3}(\mathbf{C N})_{2}{ }^{-\bullet}$, the quantitative analysis of $\sigma$ shows the polaron is more spread out at the TS state (Supporting Information Table S1), which is not obvious from the orbital picture only (Supporting Information Figure S15). We consider the rate constant for electron transfer expressed by the following equation ${ }^{81}$

$k_{\mathrm{ET}}=\kappa_{\mathrm{e}} v_{\mathrm{n}} \exp \left(\frac{-\Delta G^{\ddagger}}{R T}\right)$

where $\kappa_{\mathrm{e}}$ is the electronic factor and $v_{\mathrm{n}}$ is the nuclear frequency factor. For the present anion systems, we may safely assume that $\kappa_{\mathrm{e}}=1$, as the adiabatic parameter ${ }^{70} \mathcal{H}_{\mathrm{A}}=\frac{4 \pi H_{\mathrm{ab}}^{2} \tau_{\mathrm{L}}}{\hbar \lambda}$ is much greater than 1 , where $\tau_{\mathrm{L}}$ is the longitudinal dielectric relaxation time of the solvent $\left(\tau_{\mathrm{L}}\right.$ for THF is reported to be $1.6 \mathrm{ps}){ }^{82}$ The nuclear factor $v_{\mathrm{n}}$ typically ranges from $\sim 10^{11} \mathrm{~s}^{-1}$ to $\sim 10^{14} \mathrm{~s}^{-1}$ depending on whether the transfer is controlled by solvent friction or by a high frequency intramolecular vibration mode. ${ }^{71}$ The activation energies obtained here, $\Delta G^{\ddagger} \leq k_{\mathrm{B}} T$ do not strictly allow us to use the rate equation above as the transition state-like form of the equation assumes at least $\Delta G^{\ddagger} \geq \sim(2-3) k_{\mathrm{B}} T .^{83}$ However, we can still provide rough estimates of the rates. Adopting 
the prefactor $v_{\mathrm{n}}=k_{\mathrm{B}} T / \mathrm{h}\left(\sim 6 \times 10^{12} \mathrm{~s}^{-1}\right.$ at $\left.293 \mathrm{~K}\right)$, we obtain $k_{\mathrm{ET}} \sim 4 \times 10^{12} \mathrm{~s}^{-1}$ for $\mathbf{F}_{\mathbf{2}}(\mathbf{C N})_{2}{ }^{-\bullet}$ and $\sim 2$ $\mathrm{x} 10^{12} \mathrm{~s}^{-1}$ for $\mathbf{F}_{3}(\mathbf{C N})_{2}^{-\bullet}$. On the other hand, when solvent control is assumed, $\quad v_{\mathrm{n}}=$ $\tau_{\mathrm{L}}^{-1}\left(\lambda_{\mathrm{S}} / 16 \pi k_{\mathrm{B}} T\right)^{1 / 2}$ where $\lambda_{\mathrm{s}}$ is the solvent reorganization energy. ${ }^{70}$ This analysis only applies to $\mathbf{F}_{3}(\mathbf{C N})_{2}^{-\bullet}$ where we can estimate $\lambda_{\mathrm{s}}$ by computation (see Supporting Information Section VIII). We obtained $k_{\mathrm{ET}} \sim 5.0 \times 10^{10} \mathrm{~s}^{-1}$ for $\mathbf{F}_{3}(\mathbf{C N})_{2}{ }^{-\bullet}$. Thus in either case, the ET rate is $1-3$ orders of magnitude slower than the timescale defined by the $\mathrm{C} \equiv \mathrm{N}$ stretching frequency $\left(\sim 6 \times 10^{13} \mathrm{~s}^{-1}\right)$, consistent with the experimental observation of two distinct $\mathrm{C} \equiv \mathrm{N}$ stretching frequencies. We note that in both cases the nuclear factors themselves are well below the $\mathrm{C} \equiv \mathrm{N}$ stretching frequency.

2.5 Localization vs Delocalization It can be difficult to distinguish localization and delocalization of electronic states, especially for mixed-valence compounds, including the famous Creutz-Taube ion. ${ }^{17,18}$ The Robin and Day scheme ${ }^{84}$ divides mixed-valence compounds into three Classes. Class I is the limiting case of localization with $H_{\mathrm{ab}}=0$, in which no electron transfer occurs between the two redox sites. In Class II, an odd electron is localized with measurable electronic coupling $\left(H_{\mathrm{ab}} \neq 0\right)$. Class III is the case of delocalization, which occurs when $2 H_{\mathrm{ab}} / \lambda \geq 1$. Class II is considered to occur when $2 H_{\mathrm{ab}} / \lambda<1$. Borderline species between Class II and Class III are called Class II-III compounds. ${ }^{19}$ The classification based on $H_{\mathrm{ab}}$ and $\lambda$ is a rough estimate. Meyer and colleagues noted ${ }^{19}$ that three kinds of motions are important to distinguish localization and delocalization in mixed-valence systems; solvent, vibrational, and electronic. Based on their definitions, in Class II, the solvent and electron are localized. In Class II-III compounds, the solvent is averaged out and does not contribute to the dynamics of barrier crossing while the exchanging electron is localized, resulting in the electron transfer process 
being largely controlled by intramolecular vibrations. In Class III, the solvent and vibrations are averaged and the exchanging electron is delocalized.

We would like to apply the Robin-Day scheme to our compounds using data collected here. The above analysis shows that the electron is localized and responds to solvent motions in $\mathbf{F}_{3}(\mathbf{C N})_{2}{ }^{-\bullet}$ and thus it falls into the category of the Class II compounds. The narrow bandwidth of the $\mathrm{P}_{1}$ band in $\mathbf{F}_{2}(\mathbf{C N})_{2}{ }^{-\bullet}$ indicates solvent averaging, ${ }^{19}$ generally excluding the possibility of Class II. DFT calculations show a delocalized electron and electrochemical measurements suggests a further delocalization compared to $\mathbf{F}_{\mathbf{2}} \mathbf{C N}^{-}$. Yet, we observed two $v(C \equiv N)$ IR peaks in the more polar media, DMF and THF, which point to a transient asymmetry of the electronic distribution. These results combined point to the picture that $\mathbf{F}_{2}(\mathbf{C N})_{2}{ }^{-\bullet}$ is a Class III compound exhibiting oscillatory behavior. ${ }^{19}$ More precisely, the degree of delocalization does not change, but the distribution of charge densities changes with time. Alternatively, it might be classified as a Class II-III compound like the Creutz-Taube ion. Low energy barriers ensure that the transitions between the two sides are smooth on the ps time scale. A localized picture of the electron in $\mathbf{F}_{3}(\mathbf{C N})_{2}{ }^{-\bullet}$ indicates that the symmetry is broken when an electron is attached to these symmetric molecules. It is unclear if the symmetry is already broken before an electron attachment by nuclear and solvent fluctuations as is considered to occur in photo-induced electron transfer processes. ${ }^{85}$ A simple calculation may indicate that the symmetry gets broken after electron attachment (Supporting Information Figure S7). In either case, a lower energy barrier quickly establishes an equilibrium. In principle, the process could be probed with ultrafast IR detection coupled with pulse radiolysis, but such a capability is not currently available. We would like to note that Moneo et al. studied structurally similar oligo-( $p$ phenylene)dicarbonitriles $\left(\mathrm{Ph}_{\mathrm{n}} \mathrm{CN}_{2}, \mathrm{n}=1-4\right)$. They classified all as Class III compounds in DMF 
based on electrochemical and optical measurements. ${ }^{86}$ Our results suggest that a similar dynamic picture obtained through our studies may be applied in their longest molecule $\mathrm{Ph}_{4} \mathrm{CN}_{2}{ }_{2}$.

2.6 Implications in Conjugated Chains "Molecular wires" imply a seamless transport of an exciton and polaron over a long distance. It is however a difficult task to determine how seamless and smooth these transports are in long conjugated polymers. Some measurements find that transport is not entirely diffusion-controlled, indicating the possible existence of an energy barrier or trap in chains. Existing data indicates barriers may be more relvant. ${ }^{87-92}$ These observations raise the question: what does the potential energy surface look like in a long polymer chain? We expect that it is "smoother" in homogenous polymers such as polyfluorenes ${ }^{93}$ and polythiophenes ${ }^{94}$ than those in donor-acceptor type polymers such as F8BT $^{95}$ that have corrugated potential surfaces. However, experimental measurements of such properties in a long polymer chain are difficult. Here, by using small organic mixed-valence compounds, we have established that small activation energies $\leq k_{\mathrm{B}} T$ are present in electron movement in conjugated oligofluorenes. Based on the structural and electronic similarities, we reason that a similarly small activation energy or energy barrier is present in longer polyfluorenes and possibly in other polymers where dihedral angles are present, and that this dictates the capability of electron transport in "molecular wires." This view implied here is similar to the one by Lacroix et al. who examined theoretically the idea that mixed-valence compounds and longer conjugated polymers can be treated within the same framework. ${ }^{96}$ The estimated energy barrier is two to four times smaller than those associated with "bad" dihedral angles that we estimated in a previous study, ${ }^{47}$ further supporting the notion that such defects, if present, can hamper the transport capability significantly. 


\section{Conclusion}

We have shown that the $v(C \equiv N)$ nitrile vibration is very responsive to the degree of delocalization of electrons in anions of nitrile-substituted oligofluorenes. Measurements of a series of the mononitrile-functionalized compounds allowed us to construct a calibration curve that can be used to estimate the changes in the electronic density distributions from observed shifts in $\mathrm{C} \equiv \mathrm{N}$ frequencies. Linewidths for the $\mathrm{C} \equiv \mathrm{N}$ IR absorptions in the anions studied vary over a factor of five depending on the aryl to which the $\mathrm{C} \equiv \mathrm{N}$ is attached. While not understood yet in detail the linewidths appear to be sensitive to flexible dihedral angles. With greater understanding they may offer valuable insight into dynamic motions in the structures they are attached to. We also presented a clearer picture of the delocalization states of anions using the moment-based approach. In combination with existing theory, we developed a new method (IRCHARGE) to examine the exchanging electron behavior in the anions of dinitrile-functionalized oligofluorenes, classified as organic mixed-valence compounds. The current results show that the electron localization is not static, but can be dynamic. Physical electron localization, observed by TRIR, shows a transient symmetry breaking in these mixed-valence compounds where the bridge and charge centers are highly coupled. It is not yet clear if symmetry is broken before or after the addition of an electron, but a small activation energy of $\leq k_{\mathrm{B}} T$ quickly establishes an equilibrium. The source of the small activation energy is likely the movements of dihedral angles connecting the fluroerene units. A similar energy barrier is expected to be present in longer normal polymers where dihedral angles are present, which can dictate the limit of electron transport in "molecular wires.” The estimated energy barrier is two to four times smaller than those associated with "bad” dihedral angles, further supporting the notion that such defects can hamper the transport capability. 


\section{ASSOCIATED CONTENT}

Supporting Information. Synthetic schemes, procedures, and characterization of new compounds, determination of the polaron widths in the anions, analysis of comproportionation equilibria, discussion on the optical transitions, description of the IR-CHARGE method, information about delocalization from optical spectra, further discussion on the origin of two $v(\mathrm{C} \equiv \mathrm{N})$ IR peaks, estimation of the rate constant for solvent-controlled electron transfer, Figures S1-S15, Table S1-S2. This material is available free of charge via the Internet at htttp://pubs.acs.org.

\section{AUTHOR INFORMATION}

\section{Corresponding Author}

*Email: tmani@bnl.gov

\section{Notes}

The authors declare no competing financial interests.

\section{ACKNOWLEDGMENT}

This work, and use of the LEAF facility of the BNL Accelerator Center for Energy Research, was supported by the U.S. Department of Energy, Office of Science, Office of Basic Energy Sciences, Division of Chemical Sciences, Geosciences \& Biosciences under contract no. DESC0012704. T.M. is grateful for the support by the Goldhaber Distinguished Fellowship from Brookhaven Science Associates.

\section{REFERENCES}

(1) Clarke, T. M.; Durrant, J. R. Chem. Rev. 2010, 110, 6736. 
(2) Grozema, F. C.; van Duijnen, P. T.; Berlin, Y. A.; Ratner, M. A.; Siebbeles, L. D. A. J. Phys. Chem. B 2002, 106, 7791.

(3) Bredas, J. L.; Beljonne, D.; Coropceanu, V.; Cornil, J. Chem. Rev. 2004, 104, 4971.

(4) Takeda, N.; Asaoka, S.; Miller, J. R. J. Am. Chem. Soc. 2006, 128, 16073.

(5) Chi, C. Y.; Wegner, G. Macromol. Rapid Comm. 2005, 26, 1532.

(6) Zaikowski, L.; Kaur, P.; Gelfond, C.; Selvaggio, E.; Asaoka, S.; Wu, Q.; Chen, H. C.; Takeda, N.; Cook, A. R.; Yang, A.; Rosanelli, J.; Miller, J. R. J. Am. Chem. Soc. 2012, 134, 10852.

(7) Bakalis, J.; Cook, A. R.; Asaoka, S.; Forster, M.; Scherf, U.; Miller, J. R. J. Phys. Chem. C 2014, 118, 114.

(8) Zerbi, G.; Chierichetti, B.; Inganas, O. J. Chem. Phys. 1991, 94, 4646.

(9) Zamadar, M.; Asaoka, S.; Grills, D. C.; Miller, J. R. Nat. Commun. 2013, 4, 2818.

(10) Volk, M.; Gilch, P.; Kompa, C.; Haselsberger, R.; Harter, P.; Stockl, M.; Scherer, W.; Latzel, K.; Michel-Beyerle, M. E. J. Phys. Chem. A 2000, 104, 4984.

(11) Rubtsov, I. V.; Kang, Y. K.; Redmore, N. P.; Allen, R. M.; Zheng, J. R.; Beratan, D. N.; Therien, M. J. J. Am. Chem. Soc. 2004, 126, 5022.

(12) Barbour, L. W.; Hegadorn, M.; Asbury, J. B. J. Am. Chem. Soc. 2007, 129, 15884.

(13) Pensack, R. D.; Asbury, J. B. J. Am. Chem. Soc. 2009, 131, 15986.

(14) Yang, J. H.; Zhang, W. W.; Si, Y. B.; Zhao, Y. J. Phys. Chem. B 2012, 116, 14126.

(15) Koch, M.; Letrun, R.; Vauthey, E. J. Am. Chem. Soc. 2014, 136, 4066. 
(16) Di Nuzzo, D.; Fontanesi, C.; Jones, R.; Allard, S.; Dumsch, I.; Scherf, U.; von Hauff, E.; Schumacher, S.; Da Como, E. Nat. Commun. 2015, 6, 6460.

(17) Creutz, C.; Taube, H. J. Am. Chem. Soc. 1969, 91, 3988.

(18) Creutz, C.; Taube, H. J. Am. Chem. Soc. 1973, 95, 1086.

(19) Demadis, K. D.; Hartshorn, C. M.; Meyer, T. J. Chem. Rev. 2001, 101, 2655.

(20) Hankache, J.; Wenger, O. S. Chem. Rev. 2011, 111, 5138.

(21) Nelsen, S. F.; Ismagilov, R. F.; Trieber II, D. A. Science 1997, 278, 846.

(22) Heckmann, A.; Lambert, C. Angew. Chem. Int. Edit. 2012, 51, 326.

(23) Mani, T.; Grills, D. C.; Miller, J. R. J. Am. Chem. Soc. 2015, 137, 1136.

(24) Hosoi, H.; Mori, Y.; Masuda, Y. Chem. Lett. 1998, 177.

(25) Wishart, J. F.; Cook, A. R.; Miller, J. R. Rev. Sci. Instrum. 2004, 75, 4359.

(26) Grills, D. C.; Farrington, J. A.; Layne, B. H.; Preses, J. M.; Bernstein, H. J.;

Wishart, J. F. Rev. Sci. Instrum. 2015, 86, 044102.

(27) Frisch, M. J.; Trucks, G. W.; Schlegel, H. B.; Scuseria, G. E.; Robb, M. A.;

Cheeseman, J. R.; Scalmani, G.; Barone, V.; Mennucci, B.; Petersson, G. A.; Nakatsuji, H.;

Caricato, M.; Li, X.; Hratchian, H. P.; Izmaylov, A. F.; Bloino, J.; Zheng, G.; Sonnenberg, J. L.;

Hada, M.; Ehara, M.; Toyota, K.; Fukuda, R.; Hasegawa, J.; Ishida, M.; Nakajima, T.; Honda, Y.; Kitao, O.; Nakai, H.; Vreven, T.; Montgomery Jr., J. A.; Peralta, J. E.; Ogliaro, F.; Bearpark, M. J.; Heyd, J.; Brothers, E. N.; Kudin, K. N.; Staroverov, V. N.; Kobayashi, R.; Normand, J.; Raghavachari, K.; Rendell, A. P.; Burant, J. C.; Iyengar, S. S.; Tomasi, J.; Cossi, M.; Rega, N.; Millam, N. J.; Klene, M.; Knox, J. E.; Cross, J. B.; Bakken, V.; Adamo, C.; Jaramillo, J.;

Gomperts, R.; Stratmann, R. E.; Yazyev, O.; Austin, A. J.; Cammi, R.; Pomelli, C.; Ochterski, J. W.; Martin, R. L.; Morokuma, K.; Zakrzewski, V. G.; Voth, G. A.; Salvador, P.; Dannenberg, J. 
J.; Dapprich, S.; Daniels, A. D.; Farkas, Ö.; Foresman, J. B.; Ortiz, J. V.; Cioslowski, J.; Fox, D. J.; Gaussian, Inc.: Wallingford, CT, USA, 2009.

(28) Vydrov, O. A.; Heyd, J.; Krukau, A. V.; Scuseria, G. E. J. Chem. Phys. 2006, 125, 074106.

(29) Vydrov, O. A.; Scuseria, G. E. J. Chem. Phys. 2006, 125, 234109.

(30) Vydrov, O. A.; Scuseria, G. E.; Perdew, J. P. J. Chem. Phys. 2007, 126, 154109.

(31) Becke, A. D. J. Chem. Phys. 1993, 98, 5648.

(32) Stephens, P. J.; Devlin, F. J.; Chabalowski, C. F.; Frisch, M. J. J. Phys. Chem.

1994, 98, 11623.

(33) Breneman, C. M.; Wiberg, K. B. J. Comput. Chem. 1990, 11, 361.

(34) Grozema, F. C.; Telesca, R.; Jonkman, H. T.; Siebbeles, L. D. A.; Snijders, J. G. J. Chem. Phys. 2001, 115, 10014.

(35) Cave, R. J.; Burke, K.; Castner, E. W. J. Phys. Chem. A 2002, 106, 9294.

(36) Improta, R.; Barone, V.; Scalmani, G.; Frisch, M. J. J. Chem. Phys. 2006, 125, 0541031.

(37) Improta, R.; Scalmani, G.; Frisch, M. J.; Barone, V. J. Chem. Phys. 2007, 127, 0745041.

(38) Tomasi, J.; Mennucci, B.; Cammi, R. Chem. Rev. 2005, 105, 2999.

(39) Cave, R. J.; Edwards, S. T.; Kouzelos, J. A.; Newton, M. D. J. Phys. Chem. B 2010, 114, 14631.

(40) Subotnik, J. E.; Cave, R. J.; Steele, R. P.; Shenvi, N. J. Chem. Phys. 2009, 130.

(41) Boys, S. F. Rev. Mod. Phys. 1960, 32, 296.

(42) Subotnik, J. E.; Dutoi, A. D.; Head-Gordon, M. J. Chem. Phys. 2005, 123. 
(43) In the Gaussian function, $2(2 \ln (2))^{1 / 2} \sigma=$ FWHM.

(44) Franco, I.; Tretiak, S. J. Am. Chem. Soc. 2004, 126, 12130.

(45) Parthey, M.; Kaupp, M. Chem. Soc. Rev. 2014, 43, 5067.

(46) Cohen, A. J.; Mori-Sanchez, P.; Yang, W. T. Science 2008, 321, 792.

(47) Mani, T.; Miller, J. R. J. Phys. Chem. A 2014, 118, 9451.

(48) Renz, M.; Kaupp, M. J. Phys. Chem. A 2012, 116, 10629.

(49) Boxer, S. G. J. Phys. Chem. B 2009, 113, 2972.

(50) Levinson, N. M.; Fried, S. D.; Boxer, S. G. J. Phys. Chem. B 2012, 116, 10470.

(51) Fesser, K.; Bishop, A. R.; Campbell, D. K. Phys Rev B 1983, 27, 4804.

(52) Cave, R. J.; Newton, M. D. Chem. Phys. Lett. 1996, 249, 15.

(53) Cave, R. J.; Newton, M. D. J. Chem. Phys. 1997, 106, 9213.

(54) Hush, N. S. Coordin. Chem. Rev. 1985, 64, 135.

(55) Nelsen, S. F.; Newton, M. D. J. Phys. Chem. A 2000, 104, 10023.

(56) Subotnik, J. E.; Yeganeh, S.; Cave, R. J.; Ratner, M. A. J. Chem. Phys. 2008, 129, 244101.

(57) Bublitz, G. U.; Boxer, S. G. Annu. Rev. Phys. Chem. 1997, 48, 213.

(58) Liptay, W. Angew. Chem. Int. Edit. 1969, 8, 177.

(59) Gould, I. R.; Young, R. H.; Mueller, L. J.; Albrecht, A. C.; Farid, S. J. Am. Chem. Soc. 1994, 116, 8188.

(60) Nelsen, S. F.; Weaver, M. N.; Telo, J. P. J. Am. Chem. Soc. 2007, 129, 7036.

(61) Juchnovski, I. N.; Binev, I. G. Chem. Phys. Lett. 1971, 12, 40.

(62) Juchnovski, I. N.; Binev, I. G. J. Mol. Struct. 1971, 7, 490. 
(63) Kim, H. M.; Park, J.; Lee, Y. T.; Lim, M.; Chung, Y. K.; Kang, Y. K. J. Phys.

Chem. C 2011, 115, 22557.

(64) Grabowski, Z. R.; Rotkiewicz, K.; Rettig, W. Chem. Rev. 2003, 103, 3899.

(65) Mazur, S.; Sreekumar, C.; Schroeder, A. H. J. Am. Chem. Soc. 1976, 98, 6713.

(66) Schroeder, A. H.; Mazur, S. J. Am. Chem. Soc. 1978, 100, 7339.

(67) Ito, T.; Hamaguchi, T.; Nagino, H.; Yamaguchi, T.; Washington, J.; Kubiak, C. P. Science 1997, 277, 660.

(68) Kubiak, C. P. Inorg. Chem. 2013, 52, 5663.

(69) Hoekstra, R. M.; Telo, J. P.; Wu, Q.; Stephenson, R. M.; Nelsen, S. F.; Zink, J. I. J. Am. Chem. Soc. 2010, 132, 8825.

(70) Jortner, J.; Bixon, M. J. Chem. Phys. 1988, 88, 167.

(71) Brunschwig, B. S.; Creutz, C.; Sutin, N. Chem. Soc. Rev. 2002, 31, 168.

(72) Nelsen, S. F.; Ismagilov, R. F.; Powell, D. R. J. Am. Chem. Soc. 1997, 119, 10213.

(73) Nelsen, S. F.; Ismagilov, R. F.; Gentile, K. E.; Powell, D. R. J. Am. Chem. Soc. 1999, 121, 7108

(74) Lindeman, S. V.; Rosokha, S. V.; Sun, D. L.; Kochi, J. K. J. Am. Chem. Soc. 2002, 124, 843.

(75) Kattnig, D. R.; Mladenova, B.; Grampp, G.; Kaiser, C.; Heckmann, A.; Lambert, C. J. Phys. Chem. C 2009, 113, 2983.

(76) Zhou, G.; Baumgarten, M.; Mullen, K. J. Am. Chem. Soc. 2007, 129, 12211.

(77) Lambert, C.; Nöll, G. J. Am. Chem. Soc. 1999, 121, 8434.

(78) Coropceanu, V.; Malagoli, M.; Andre, J. M.; Bredas, J. L. J. Am. Chem. Soc. 2002, 124, 10519. 
(79) Fratiloiu, S.; Grozerna, F. C.; Koizumi, Y.; Seki, S.; Saeki, A.; Tagawa, S.; Dudek, S. P.; Siebbeles, L. D. A. J. Phys. Chem. B 2006, 110, 5984.

(80) Although an excess charge is considered fully delocalized in a system, it could still exhibit IR absorption characteristic of localization as discussed in refs 19 and 71.

(81) Sutin, N. Accounts Chem. Res. 1982, 15, 275.

(82) Grampp, G.; Rasmussen, K. Phys. Chem. Chem. Phys. 2002, 4, 5546.

(83) Weaver, M. J. Chem. Rev. 1992, 92, 463.

(84) Robin, M. B.; Day, P. In Advances in Inorganic Chemistry and Radiochemistry; Emeléus, H. J., Sharpe, A. G., Eds.; Academic Press: 1968; Vol. Volume 10, p 247.

(85) Vauthey, E. Chemphyschem 2012, 13, 2001.

(86) Moneo, A.; Carvalho, M. F. N. N.; Telo, J. P. J. Phys. Org. Chem. 2012, 25, 559.

(87) Grozema, F. C.; Hoofman, R. J. O. M.; Candeias, L. P.; de Haas, M. P.; Warman, J. M.; Siebbeles, L. D. A. J. Phys. Chem. A 2003, 107, 5976.

(88) Prins, P.; Grozema, F. C.; Galbrecht, F.; Scherf, U.; Siebbeles, L. D. A. J. Phys. Chem. C 2007, 111, 11104.

(89) Asaoka, S.; Takeda, N.; Iyoda, T.; Cook, A. R.; Miller, J. R. J. Am. Chem. Soc. 2008, 130, 11912.

(90) Shibano, Y.; Imahori, H.; Sreearunothai, P.; Cook, A. R.; Miller, J. R. J. Phys. Chem. Lett. 2010, 1, 1492.

(91) Grozema, F. C.; Siebbeles, L. D. A. J. Phys. Chem. Lett. 2011, 2, 2951.

(92) Bird, M. J.; Reid, O. G.; Cook, A. R.; Asaoka, S.; Shibano, Y.; Imahori, H.; Rumbles, G.; Miller, J. R. J. Phys. Chem. C 2014, 118, 6100.

(93) Scherf, U.; List, E. J. W. Adv. Mater. 2002, 14, 477. 
(94) McCullough, R. D. Adv. Mater. 1998, 10, 93.

(95) Duan, C. H.; Huang, F.; Cao, Y. J. Mater. Chem. 2012, 22, 10416.

(96) Lacroix, J. C.; Chane-Ching, K. I.; Maquere, F.; Maurel, F. J. Am. Chem. Soc. 2006, $128,7264$. 


\section{TOC GRAPHICS}

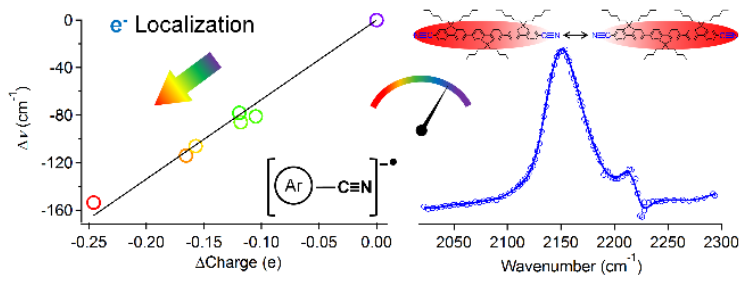

